\title{
ON THE QUANTUM MECHANICS OF SUPERMEMBRANES
}

\author{
B. de WIT \\ Institute for Theoretical Physics, University of Utrecht, POB 80.006, 3508 TA Utrecht, \\ The Netherlands \\ J. HOPPE and H. NICOLAI* \\ Institut für Theoretische Physik, Universität Karlsruhe, POB 6380, D 7500 Karlsruhe, FRG
}

Received 11 April 1988

\begin{abstract}
We study the quantum-mechanical properties of a supermembrane and examine the nature of its ground state. A supersymmetric gauge theory of area-preserving transformations provides a convenient framework for this study. The supermembrane can be viewed as a limiting case of a class of models in supersymmetric quantum mechanics. Its mass does not depend on the zero modes and vanishes only if the wave function is a singlet under supersymmetry transformations of the nonzero modes. We exhibit the complexity of the supermembrane ground state and examine various truncations of these models. None of these truncations has massless states.
\end{abstract}

\section{Introduction}

Some time ago an action for a membrane moving in a $d$-dimensional space-time was formulated, which is invariant under super-Poincaré transformations [1]. It is expressed in terms of the membrane coordinates $X^{\mu}(\zeta)$ and a set of anticommuting coordinates $\theta(\zeta)$, transforming as a $d$-dimensional vector and spinor, respectively; the parameters $\zeta^{i}(i=0,1,2)$ parametrize the world tube swept out by the membrane in space-time. As is well-known, similar actions exist for the superparticle, the superstring, as well as higher-extended objects (" $p$-branes") [1-4], and they are all characterized by the presence of a local (i.e., $\zeta$-dependent) fermionic symmetry. This invariance requires the existence of a closed superspace form [5], appearing in the action in the form of a Wess-Zumino-Witten term, which is only possible for a specific number of space-time dimensions. Therefore, the supermembrane action can only be formulated in $d=4,5,7$ and 11 dimensional space-times.

An intriguing result found in [1] is that a supermembrane can propagate in a curved superspace. In particular for $d=11$, the membrane can couple consistently (i.e., without affecting the local fermionic symmetry) to a $d=11$ supergravity background. Guided by the experience in string theory this result has been interpreted as an indication that the ground state of the supermembrane should be

*Address from May 1, 1988: II. Institut für Theoretische Physik, Universität Hamburg. 
degenerate and constitute the states of a massless $d=11$ supergravity multiplet. In attempts to study this question the quantum fluctuations have been analyzed about solutions of the classical membrane equations [6,7]. While the vacuum energy of these fluctuations vanishes for the solution considered in [6], it did not vanish for the solution described in [7], and neither did it constitute an integer as it does in the case of the string [8] (for the (open) bosonic membrane such a calculation was first undertaken in [9]). On the other hand, heuristic arguments were presented in [10], based on the vanishing of the vacuum energy for fluctuations about a solution with residual supersymmetry, which support the conjecture that the ground state has the structure of a massless $d=11$ supermultiplet.

In this paper we will study the quantum mechanics of a supermembrane in more detail in the hope of constructing the ground-state wave function. We present an alternative formulation of the membrane as a gauge theory of the area-preserving transformations of the membrane surface. Here we are inspired by the fact that these transformations are the residual invariance of a relativistic membrane theory when quantized in the light-cone gauge [11]. It is possible to consider truncations of this gauge theory by truncating the infinite harmonic expansion of the membrane coordinates. At least for membranes with the topology of a sphere this can be done in such a way that the supersymmetry remains preserved. The group of area-preserving transformations is thereby reduced to $\mathrm{SU}(N){ }^{\star}$ These truncations lead to a class of matrix models in supersymmetric quantum mechanics [13,14], which turn out to coincide with the models that have been presented in [15]. A priori, three different types of membrane ground states are possible. One possibility is that the ground state is a singlet under supersymmetry, which is thus annihilated by the supersymmetry charges. By virtue of the anticommutation relation which expresses the hamiltonian as the square of these charges, this ground state should be massless. However, this situation is not possible for the supermembrane: it follows from the explicit expression for the hamiltonian that all wave functions have an obvious degeneracy associated with the fermionic zero modes. Therefore the ground state must be degenerate and constitute a supermultiplet. There are then two possibilities. One is that the ground state is a massless supermultiplet, consisting of $2^{7}$ bosonic and $2^{7}$ fermionic states, in which case the supercharges associated with the nonzero modes must annihilate the ground-state wave functions. If this is not the case one has a massive supermultiplet. The ground-state degeneracy is then enormous, as a massive supermultiplet contains $2^{15}$ bosonic and $2^{15}$ fermionic states.

We restrict ourselves to supermembranes that move in a trivial space-time. Hence we consider no compactification as in [6] and neither do we study the possibility of membranes moving in nontrivial space-times such as in [16]. This means, in particular, that our considerations have no bearing on the results described in [16],

\footnotetext{
* This idea goes back to Goldstone [11]. The relation between SU(N) and the group of area-preserving transformations was exhibited in [12]
} 
where the existence of infinitely many massless states of the supermembrane compactified to $\mathrm{AdS}_{4} \times \mathrm{S}^{7}$ was demonstrated in a small-fluctuation analysis. Our work shows that the ground-state wave function of a supermembrane has a high degree of complexity. For instance, it is not possible for a massless ground state that the wave function factorizes into a bosonic and a fermionic part, if one of these factors is rotationally invariant. This is a distinct difference with the wave function for the superstring ground state. We then study the restrictions imposed by rotational invariance for the total ground-state wave function, but, unfortunately, this does not lead to useful simplifications. Although the condition that the wave function vanishes under the action of the supersymmetry charges has solutions, these solutions tend to be not square-integrable. This we demonstrate in a $G_{2^{-}}$ invariant truncation of the theory. We also consider a supermembrane propagating in a 4-dimensional space-time in a truncation where the group of area-preserving transformations is reduced to SU(2). Assuming that the wave function tends to zero at spatial infinity, we show that the energy of the supermembrane is lower than that of its bosonic version, but there is no solution with zero mass. However, the complexity of this problem makes it hard to reach a firm conclusion concerning the existence of massless solutions in the general case. We should also emphasize that, while the supersymmetric matrix models are well defined, it is not clear what will happen in the limit where the gauge group approaches the full infinite-dimensional group of area-preserving transformations. As is well-known, in quantum-mechanical systems based on an infinite number of degrees of freedom, degenerate ground states are not always contained in the same Hilbert space; this aspect is of immediate importance for possible applications of supermembrane theories. Also, while the models based on SU(N) yield, in the limit $N \rightarrow \infty$, the full group of area-preserving transformations corresponding to a membrane with the topology of a sphere, a corresponding result for other membrane topologies is not known.

In sect. 2 we start by formulating the membrane action in the light-cone gauge, emphasizing the role played by the area-preserving transformations. We introduce a gauge theory of these transformations, and verify the supersymmetry algebra. In sect. 3 we review the truncation to the finite-dimensional matrix models and discuss some properties of area-preserving transformations. Then, in sect. 4 , we discuss attempts to solve the equations for the ground-state wave function of the supermembrane and demonstrate the absence of a massless ground state in two different truncations. In an appendix we analyze the implications of $\mathrm{SO}(9)$ invariance for a general wave function.

\section{Lightcone formulation of the supermembrane}

The starting point of this section is the lagrangian

$$
\mathscr{L}=-\sqrt{-g(X, \hat{\theta})}-\varepsilon^{i j k}\left[\frac{1}{2} \partial_{i} X^{\mu}\left(\partial_{j} X^{\nu}+\bar{\theta} \Gamma^{\nu} \partial_{j} \theta\right)+\frac{1}{6} \bar{\theta} \Gamma^{\mu} \partial_{i} \theta \bar{\theta} \Gamma^{\nu} \partial_{j} \theta\right] \bar{\theta} \Gamma_{\mu \nu} \partial_{k} \theta,
$$


where $X^{\mu}(\zeta)$ and $\theta(\zeta)$ denote the superspace coordinates of the membrane parametrized in terms of world-tube parameters $\zeta^{i}(i=0,1,2)$. The metric $g_{i j}(X, \theta)$ is the induced metric on the world tube,

$$
g_{i j}=E_{i}^{\mu} E_{j}^{\nu} \eta_{\mu \nu},
$$

where $E_{i}^{\mu}$ are certain supervielbein components tangential to the world tube, defined by

$$
E_{i}^{\mu}=\partial_{i} X^{\mu}+\bar{\theta} \Gamma^{\mu} \partial_{i} \theta
$$

and $\eta_{\mu \nu}$ is the flat $d=11$ Minkowski metric. It is easy to see that $E_{i}^{\mu}$ is invariant under space-time supersymmetry transformations

$$
\delta \theta=\varepsilon, \quad \delta X^{\mu}=-\bar{\varepsilon} \Gamma^{\mu} \theta .
$$

In fact this transformation also leaves the lagrangian (2.1) invariant (up to a total divergence) provided the following gamma matrix identity is satisfied

$$
\bar{\psi}_{[1} \Gamma^{\mu} \psi_{2} \bar{\psi}_{3} \Gamma_{\mu \nu} \psi_{4]}=0 \text {, }
$$

where we antisymmetrize over four arbitrary spinors $\psi_{1}-\psi_{4}$. This identity only holds in $d=4,5,7$ and 11 space-time dimensions. In this paper we mainly restrict ourselves to $d=11$, but this restriction is not important for the analysis to be presented below.

The field equations corresponding to the lagrangian (2.1) take the form

$$
\begin{array}{r}
\partial_{i}\left(\sqrt{-g} g^{i j} E_{j}^{\mu}\right)-\varepsilon^{i j k} E_{i}^{\nu} \partial_{j} \bar{\theta} \Gamma_{\nu}^{\mu} \partial_{k} \theta=0, \\
(\mathbf{1}+\Gamma) g^{i j} E_{i} \partial_{j} \theta=0,
\end{array}
$$

where $\Gamma$ is defined by

$$
\Gamma=\frac{\varepsilon^{i j k}}{6 \sqrt{-g}} E_{i}^{\mu} E_{j}^{\nu} E_{k}^{\rho} \Gamma_{\mu \nu \rho} .
$$

We note two important identities for $\Gamma$,

$$
\Gamma^{2}=1, \quad \Gamma E_{i}^{\prime}=E_{i} \Gamma=g_{i j} \frac{\varepsilon^{j k l}}{2 \sqrt{-g}} E_{k}^{\mu} E_{l}^{\nu} \Gamma_{\mu \nu} .
$$

The lagrangian (2.1) is manifestly invariant under reparametrizations of the world-tube coordinates $\zeta^{i}$. It is also invariant under a local fermionic symmetry 
generated by

$$
\theta=(\mathbf{1}-\Gamma) \kappa, \quad \delta X^{\mu}=\bar{\kappa}(\mathbf{1}-\Gamma) \Gamma^{\mu} \boldsymbol{\theta},
$$

where $\kappa$ is an arbitrary $\zeta$-dependent spinor. Observe that $\kappa$ is always multiplied by the projection operator $(1-\Gamma)$.

Of particular importance is the supersymmetry current associated with the transformations (2.4). It reads

$$
\begin{aligned}
J^{i}= & -2 \sqrt{-g} g^{i j} B_{j} \theta \\
& -\varepsilon^{i j k}\left\{E_{j}^{\mu} E_{k}^{\nu} \Gamma_{\mu \nu} \theta+\frac{4}{3}\left[\Gamma^{\nu} \theta\left(\bar{\theta} \Gamma_{\mu \nu} \partial_{j} \theta\right)+\Gamma_{\mu \nu} \theta\left(\bar{\theta} \Gamma^{\nu} \partial_{j} \theta\right)\right]\left[E_{k}^{\mu}-\frac{2}{5} \bar{\theta} \Gamma^{\mu} \partial_{k} \theta\right]\right\} .
\end{aligned}
$$

As one can verify straightforwardly, this current is conserved by virtue of the field equations (2.6) and (2.7), provided the identity (2.5) holds.

In order to pass to the light-cone gauge we choose light-cone coordinates

$$
X^{ \pm}=\sqrt{\frac{1}{2}}\left(X^{10} \pm X^{0}\right)
$$

Transverse coordinates will be denoted by $X^{a}(\zeta)(a=1, \ldots, 9)$. For the gamma matrices we make a similar decomposition,

$$
\gamma_{ \pm}=\sqrt{\frac{1}{2}}\left(\Gamma^{10} \pm \Gamma^{0}\right), \quad \gamma_{a}=\Gamma_{a}, \quad(a=1, \ldots, 9),
$$

so that $\left\{\gamma_{+}, \gamma_{-}\right\}=21, \gamma_{+}^{2}=\gamma_{-}^{2}=0,\left\{\gamma_{ \pm}, \gamma_{a}\right\}=0$. Furthermore we change notation and denote the parameters $\zeta^{i}$ by

$$
\left(\zeta^{0}, \zeta^{r}\right) \rightarrow\left(\tau, \sigma^{r}\right), \quad(r=1,2) .
$$

By a suitable reparametrization we now choose

$$
X^{+}(\zeta)=X^{+}(0)+\tau
$$

so that $\partial_{i} X^{+}=\delta_{i 0}$. Furthermore we use the local fermionic symmetry to impose the gauge condition

$$
\gamma_{+} \theta=0
$$

With these gauge conditions we obtain the following result for the components of the induced metric

$$
\begin{aligned}
& g_{r s} \equiv \bar{g}_{r s}=\partial_{r} X \cdot \partial_{s} X \\
& g_{0 r} \equiv u_{r}=\partial_{r} X^{-}+\partial_{0} X \cdot \partial_{r} X+\bar{\theta} \gamma_{-} \partial_{r} \theta \\
& g_{00}=2 \partial_{0} X^{-}+\left(\partial_{0} X\right)^{2}+2 \bar{\theta} \gamma_{-} \partial_{0} \theta
\end{aligned}
$$


while the determinant of the metric can now be written as

$$
g \equiv \operatorname{det} g=-\Delta \bar{g},
$$

where

$$
\Delta=-g_{00}+u_{r} \bar{g}^{r s} u_{s}, \quad \bar{g}^{r s} g_{s t}=\delta_{t}^{r}, \quad \bar{g} \equiv \operatorname{det} \bar{g}
$$

After imposing the gauge conditions (2.15) and (2.16) the lagrangian and supercharge densities take the form (using $\varepsilon^{0 r s}=-\varepsilon^{r s}, g^{00}=-\Delta^{-1}, g^{0 r}=\Delta^{-1} \bar{g}^{r s} u_{s}$ )

$$
\begin{aligned}
\mathscr{L} & =-\sqrt{\bar{g} \Delta}+\varepsilon^{r s} \partial_{r} X^{a} \bar{\theta} \gamma_{-} \gamma_{a} \partial_{s} \theta \\
J^{0} & =2 \sqrt{\frac{\bar{g}}{\Delta}}\left[\left(\partial_{0} X^{a}-u_{r} \bar{g}^{r s} \partial_{s} X^{a}\right) \gamma_{a}+\gamma_{-}\right] \theta+\varepsilon^{r s} \partial_{r} X^{a} \partial_{s} X^{b} \gamma_{a b} \theta
\end{aligned}
$$

In order to write down the corresponding hamiltonian density, we first determine the canonical momenta $\boldsymbol{P}, \boldsymbol{P}^{+}$and $S$ conjugate to $\boldsymbol{X}, X^{-}$and $\theta$, respectively. They are

$$
\begin{aligned}
P & \equiv \frac{\partial \mathscr{L}}{\partial\left(\partial_{0} X\right)}=\sqrt{\frac{\bar{g}}{\Delta}}\left(\partial_{0} X-u_{r} \bar{g}^{r s} \partial_{s} X\right), \\
P^{+} & \equiv \frac{\partial \mathscr{L}}{\partial\left(\partial_{0} X^{-}\right)}=\sqrt{\frac{\bar{g}}{\Delta}}, \\
S & \equiv \frac{\partial \mathscr{L}}{\partial_{L}\left(\partial_{0} \bar{\theta}\right)}=-\sqrt{\frac{\bar{g}}{\Delta}} \gamma_{-} \theta,
\end{aligned}
$$

where $\partial_{L}$ denotes the left derivative. The hamiltonian density then takes the simple form

$$
\begin{aligned}
\mathscr{H} \equiv \boldsymbol{P} \cdot \partial_{0} \boldsymbol{X}+P^{+} \partial_{0} X^{-}+\bar{S} \partial_{0} \theta-\mathscr{L} \\
=\frac{\boldsymbol{P}^{2}+\bar{g}}{2 P^{+}}-\varepsilon^{r s} \partial_{r} X^{a} \bar{\theta} \gamma_{-} \gamma_{a} \partial_{s} \theta
\end{aligned}
$$

The bosonic part of this expression was first found by Goldstone [11] (see also [12]), while its superextension was derived in [17].

One easily verifies that there are two primary constraints

$$
\begin{aligned}
\phi_{r} & =\boldsymbol{P} \cdot \partial_{r} X+P^{+} \partial_{r} X^{-}+\bar{S} \partial_{r} \theta \approx 0, \\
\chi & =S+P^{+} \gamma_{-} \theta \approx 0,
\end{aligned}
$$


where $\approx 0$ indicates that the constraints are "weakly zero", so that they may have nonzero Poisson brackets with the phase-space variables. We recall that the time (i.e., $\tau$ ) evolution in phase space is governed by the "total" hamiltonian [18])

$$
H_{\mathrm{T}}=\int \mathrm{d}^{2} \boldsymbol{\sigma}\left\{\mathscr{H}+c^{r} \phi_{r}+\bar{d} \chi\right\},
$$

where $c^{r}$ and $d$ are Lagrange multipliers. One can easily verify that there are no secondary constraints at this point.

The gauge conditions (2.15) and (2.16) are still invariant under $\tau$-dependent reparametrizations of $\sigma^{r}$

$$
\sigma^{r} \rightarrow \sigma^{r}+\xi^{r}(\tau, \sigma) .
$$

Under such infinitesimal reparametrizations $u^{r}$ changes into $u^{r}-\partial_{0} \xi^{r}+\partial_{s} \xi^{r} u^{s}-$ $\xi^{s} \partial_{s} u^{r}$, which shows that one can impose yet another gauge condition, namely

$$
u^{r}=0 \text {. }
$$

In this gauge it follows that $c^{r}=0$ according to the Hamilton equations corresponding to (2.26), so that $\partial_{0} P^{+}=0$. Because $P^{+}$transforms as a density under reparametrizations, it may be adjusted to a constant times some density $w(\sigma)$,

$$
P^{+}=P_{0}^{+} w(\sigma),
$$

where we will normalize $w(\sigma)$ according to

$$
\int \mathrm{d}^{2} \sigma w(\sigma)=1
$$

Therefore the constant $P_{0}^{+}$represents the membrane momentum in the direction associated with the coordinate $X^{-}$,

$$
P_{0}^{+}=\int \mathrm{d}^{2} \sigma P^{+}
$$

The other momentum components are given by the integrals over $\boldsymbol{P}$ and $-\mathscr{H}$,

$$
\boldsymbol{P}_{0}=\int \mathrm{d}^{2} \boldsymbol{\sigma} \boldsymbol{P}, \quad \boldsymbol{P}_{0}^{-}=-\int \mathrm{d}^{2} \boldsymbol{\sigma} \mathscr{H} .
$$

Hence the mass $\mathscr{M}$ of the membrane is given by

$$
\mathscr{M}^{2}=\int \mathrm{d}^{2} \boldsymbol{\sigma}\left\{\frac{\left[\boldsymbol{P}^{2}\right]^{\prime}+\bar{g}}{w}-2 P_{0}^{+} \varepsilon^{r s} \partial_{r} X^{a} \bar{\theta} \gamma_{-} \gamma_{a} \partial_{s} \theta\right\},
$$


where the notation $\left[\boldsymbol{P}^{2}\right]^{\prime}$ indicates that we are excluding the zero mode $\boldsymbol{P}=\boldsymbol{P}_{0} w(\boldsymbol{\sigma})$ from the integrand. On the other hand, we observe that the zero modes $X_{0}$ and $\theta_{0}$, defined by

$$
\boldsymbol{X}_{0}=\int \mathrm{d}^{2} \sigma w(\sigma) \boldsymbol{X}, \quad \theta_{0}=\int \mathrm{d}^{2} \boldsymbol{\sigma} w(\sigma) \theta
$$

do not appear in the equation for $\mathscr{M}^{2}$ either, at least if the membrane coordinates are single-valued functions of $\sigma^{r}$, which is the case if space-time is not compactified, or, for open membranes, if one assumes appropriate boundary conditions. The absence of $\boldsymbol{X}_{0}$, which is just the center-of-mass coordinate of the membrane, is rather obvious. The fact that $\mathscr{M}^{2}$ does not depend on $\theta_{0}$ will play an extremely important role later on.

The coordinate $X^{-}$no longer appears explicitly in (2.33) and is determined by the gauge condition (2.28), or, equivalently, by the constraint (2.24) after imposing the gauge condition (2.29). The relevant formula is

$$
\partial_{r} X^{-}=-\partial_{0} X \cdot \partial_{r} X-\bar{\theta} \gamma_{-} \partial_{r} \theta
$$

Because $X^{-}$must be a globally defined function of $\sigma^{r}$ this requires that

$$
\oint\left(\partial_{0} X \cdot \partial_{r} X+\bar{\theta} \gamma_{-} \partial_{r} \theta\right) \mathrm{d} \sigma^{r}=0
$$

for any closed curve on the membrane. Locally this condition implies

$$
\varepsilon^{r s}\left(\partial_{r} \partial_{0} \boldsymbol{X} \cdot \partial_{s} \boldsymbol{X}+\partial_{r} \bar{\theta} \gamma_{-} \partial_{s} \theta\right)=0
$$

Observe that, when space-time is not compactified so that $\boldsymbol{X}$ and $\boldsymbol{\theta}$ are single-valued functions of $\sigma$, only the condition (2.37) is relevant.

The gauge conditions adopted above leave a residual reparametrization invariance consisting of time-independent area-preserving transformations. Infinitesimal transformations of this kind leave (2.29) invariant, and are thus defined by

$$
\sigma^{r} \rightarrow \sigma^{r}+\xi^{r}(\sigma) \quad \text { with } \quad \partial_{r}\left(w(\sigma) \xi^{r}(\sigma)\right)=0
$$

There exists an alternative formulation of the membrane theory, which emphasizes area-preserving reparametrizations from the start. Locally the area-preserving transformations can be written as

$$
\xi^{r}(\sigma)=\frac{\varepsilon^{r s}}{w(\sigma)} \partial_{s} \xi(\sigma) .
$$

If the membrane is topologically nontrivial, i.e. if the membrane surface has handles 
so that it contains uncontractible curves, then $\xi^{r}(\sigma)$ and $\xi(\sigma)$ will not necessarily be globally defined. However, we will restrict ourselves to the subgroup generated by functions $\xi(\sigma)$ that are globally defined. It is then convenient to introduce a Lie bracket of any two functions $A(\sigma)$ and $B(\sigma)$ by

$$
\{A, B\} \equiv \frac{\varepsilon^{r_{s}}}{w(\sigma)} \partial_{r} A(\sigma) \partial_{s} B(\sigma)
$$

which is antisymmetric in $A$ and $B$ and satisfies the Jacobi identity $\{A,\{B, C\}\}+$ $\{B,\{C, A\}\}+\{C,\{A, B\}\}=0$. Using this bracket, infinitesimal area-preserving reparametrizations act on $X^{a}$ and $\theta$ according to

$$
\delta X^{a}=\left\{\xi, X^{a}\right\}, \quad \delta \theta=\{\xi, \theta\}
$$

Now let us introduce a gauge field $\omega$ associated with time-dependent reparametrizations, transforming as

$$
\delta \omega=\partial_{0} \xi+\{\xi, \omega\},
$$

and corresponding covariant derivatives

$$
D_{0} X^{a}=\partial_{0} X^{a}-\left\{\omega, X^{a}\right\}, \quad D_{0} \theta=\partial_{0} \theta-\{\omega, \theta\} .
$$

The following lagrangian density is then manifestly gauge invariant under the transformations (2.41) and (2.42),

$$
w^{-1} \mathscr{L}=\frac{1}{2}\left(D_{0} X\right)^{2}+\bar{\theta} \gamma_{-} D_{0} \theta-\frac{1}{4}\left(\left\{X^{a}, X^{b}\right\}\right)^{2}+\bar{\theta} \gamma_{-} \gamma_{a}\left\{X^{a}, \theta\right\}
$$

as well as under space-time supersymmetry transformations given by

$$
\begin{aligned}
\delta X^{a} & =-2 \bar{\varepsilon} \gamma^{a} \theta \\
\delta \theta & =\frac{1}{2} \gamma_{+}\left(D_{0} X^{a} \gamma_{a}+\gamma_{-}\right) \varepsilon+\frac{1}{4}\left\{X^{a}, X^{b}\right\} \gamma_{+} \gamma_{a b} \varepsilon \\
\delta \omega & =-2 \bar{\varepsilon} \theta
\end{aligned}
$$

The supercharge density associated with the transformations (2.45), equals

$$
J^{0}=w\left[2\left(D_{0} X^{a} \gamma_{a}+\gamma_{-}\right)+\left\{X^{a}, X^{b}\right\} \gamma_{a b}\right] \theta
$$

In the gauge $\omega=0$ the latter result coincides with the charge density obtained previously (cf. (2.21) after imposing the gauge conditions (2.28) and (2.29)). To see 
that the supersymmetry transformations are associated with space-time, one may evaluate the supersymmetry commutator on $X^{a}$ :

$$
\left[\delta\left(\varepsilon_{1}\right), \delta\left(\varepsilon_{2}\right)\right] X^{a}=-2 \bar{\varepsilon}_{2} \gamma_{+} \varepsilon_{1} D_{0} X^{a}-2 \bar{\varepsilon}_{2} \gamma^{a} \varepsilon_{1}+\left\{\xi, X^{a}\right\}
$$

where, on the right-hand side, we distinguish a $\tau$-translation generated by $D_{0}$ (which, as we know, is related to a translation of the membrane coordinate $X^{+}$), a translation of $X^{a}$ and an $X$-dependent area-preserving gauge transformation with parameter $\xi=2 \bar{\varepsilon}_{2} \gamma^{b} \gamma_{+} \varepsilon_{1} X^{b}$. In order to verify that the bosonic and fermionic degrees of freedom balance in the path integral associated with (2.44), one may impose a gauge condition $\omega=0$, which leads to a (free) fermionic complex ghost field. Altogether one then counts 9 bosonic and $16+2=18$ (real) fermionic field components.

To establish full equivalence of (2.44) with the membrane lagrangian, we implement the gauge $\omega=0$ and introduce canonical momenta $\boldsymbol{P}$ and $S$ associated with $\boldsymbol{X}$ and $\theta$,

$$
\boldsymbol{P}=w \partial_{0} \boldsymbol{X}, \quad S=-w \gamma_{-} \theta
$$

The hamiltonian is then

$$
\begin{aligned}
H & \equiv \int \mathrm{d}^{2} \sigma\left\{\boldsymbol{P} \cdot \partial_{0} \boldsymbol{X}+\bar{S} \partial_{0} \theta-\mathscr{L}\right\} \\
& =\frac{1}{2} \int \mathrm{d}^{2} \sigma\left\{w^{-1} \boldsymbol{P}^{2}+\frac{1}{2} w\left(\left\{X^{a}, X^{b}\right\}\right)^{2}-2 w \bar{\theta} \gamma_{-} \gamma_{a}\left\{X^{a}, \theta\right\}\right\},
\end{aligned}
$$

so that, after dropping the zero-mode $\boldsymbol{P}_{0}, 2 H$ coincides with eq. (2.33) for the membrane mass $\mathscr{M}$, provided $\theta$ is rescaled by a factor $\sqrt{P_{0}^{+}}$(to make the comparison, use that $\left.\left(\left\{X^{a}, X^{b}\right\}\right)^{2}=2 w^{-2} \bar{g}\right)$.

Furthermore, the field equation for $\omega$ leads to the constraint

$$
\varphi \equiv\left\{\partial_{0} \boldsymbol{X}, \boldsymbol{X}\right\}+\left\{\bar{\theta} \gamma_{-}, \theta\right\} \approx 0
$$

or, in phase-space variables,

$$
\varphi=\left\{w^{-1} \boldsymbol{P}, \cdot \boldsymbol{X}\right\}+\left\{w^{-1} \bar{S}, \theta\right\} \approx 0
$$

This constraint is just (2.37), and we have thus established the equivalence of (2.44) with the initial lagrangian (2.1). The quantity $\varphi$ is the "current" that couples to the gauge field $\omega$, so it is obvious that $w \varphi$ represents the charge density associated with the area-preserving transformations. In addition there is the usual second-class constraint that expresses the fermionic momentum $S$ into $\theta$. 
The Dirac brackets for the theory above are derived by standard methods and read

$$
\begin{aligned}
\left(X^{a}(\sigma), P^{b}\left(\sigma^{\prime}\right)\right)_{\mathrm{DB}} & =\delta^{a b} \delta^{2}\left(\sigma-\sigma^{\prime}\right) \\
\left(\theta_{a}(\sigma), \bar{\theta}_{\beta}\left(\sigma^{\prime}\right)\right)_{\mathrm{DB}} & =\frac{1}{4 w}\left(\gamma_{+}\right)_{\alpha \beta} \delta^{2}\left(\sigma-\sigma^{\prime}\right) .
\end{aligned}
$$

It is now possible to verify the full $d=11$ supersymmetry algebra. Decomposing the supersymmetry charges into two independent $\mathrm{SO}(9)$ spinors according to

$$
Q=Q^{+}+Q^{-}=\int \mathrm{d}^{2} \sigma J^{0}
$$

where $J^{0}$ is given in (2.46) and $Q^{ \pm} \equiv \frac{1}{2} \gamma_{ \pm} \gamma_{\mp} Q$, we find the expressions

$$
\begin{aligned}
& Q^{+}=\int \mathrm{d}^{2} \boldsymbol{\sigma}\left(2 P^{a} \gamma_{a}+w\left\{X^{a}, X^{b}\right\} \gamma_{a b}\right) \theta, \\
& Q^{-}=-2 \int \mathrm{d}^{2} \boldsymbol{\sigma} S=2 \gamma_{-} \theta_{0} .
\end{aligned}
$$

Observe that $Q^{-}$acts only on the fermionic zero-modes $\theta_{0}$, which, as we have pointed out before, do not appear in the expressions for the hamiltonian and the membrane mass.

It is now a straightforward exercise to determine the Dirac brackets for the supercharges. The result takes the following form

$$
\begin{aligned}
\left(Q_{\alpha}^{-}, \bar{Q}_{\beta}^{-}\right)_{\mathrm{DB}}= & -2\left(\gamma_{-}\right)_{\alpha \beta}, \\
\left(Q_{\alpha}^{+}, \bar{Q}_{\beta}^{+}\right)_{\mathrm{DB}}= & 2\left(\gamma_{+}\right)_{\alpha \beta} H-2\left(\gamma_{a} \gamma_{+}\right)_{\alpha \beta} \int \mathrm{d}^{2} \sigma w \varphi X^{a} \\
& +\left(\gamma^{a} \gamma_{+}\right)_{\alpha \beta} \int \mathrm{d}^{2} \sigma \partial_{r} S_{a}^{r}+\left(\gamma^{a b c d} \gamma_{+}\right)_{\alpha \beta} \int \mathrm{d}^{2} \sigma \partial_{r} S_{a b c d}^{r}, \\
\left(Q_{\alpha}^{+}, \bar{Q}_{\beta}^{-}\right)_{\mathrm{DB}}= & -\left(\gamma_{a} \gamma_{+} \gamma_{-}\right)_{\alpha \beta} P_{0}^{a}+\left(\gamma^{a b} \gamma_{+} \gamma_{-}\right)_{\alpha \beta} \int \mathrm{d}^{2} \alpha \partial_{r} S_{a b}^{r},
\end{aligned}
$$

where the surface terms, given by

$$
\begin{aligned}
S_{a}^{r} & =\varepsilon^{r s}\left\{2 w^{-1} X_{a} P^{b} \partial_{s} X^{b}+2 X_{a} \bar{\theta} \gamma_{-} \partial_{s} \theta-\frac{3}{8} X^{b} \partial_{s}\left(\bar{\theta} \gamma_{-} \gamma_{a b} \theta\right)\right\}, \\
S_{a b c d}^{r} & =\frac{1}{24} \varepsilon^{r s} X_{[a} \partial_{s}\left(\bar{\theta} \gamma_{-} \gamma_{b c d]} \theta\right), \\
S_{a b}^{r} & =-\frac{1}{2} \varepsilon^{r s} X_{[a} \partial_{s} X_{b]},
\end{aligned}
$$


can contribute only if the membrane coordinates and momenta are not single-valued.

It is useful to separately consider the zero-mode contributions to $Q^{+}$, which define a conserved charge $Q^{+(0)}$. It reads

$$
Q^{+(0)}=2 P_{0}^{a} \gamma_{a} \theta_{0}
$$

Together, $Q^{+(0)}$ and $Q^{-}$generate the algebra

$$
\begin{gathered}
\left(Q_{\alpha}^{-}, \bar{Q}_{\beta}^{-}\right)_{\mathrm{DB}}=-2\left(\gamma_{-}\right)_{\alpha \beta}, \\
\left(Q_{\alpha}^{+(0)}, \bar{Q}_{\beta}^{+(0)}\right)_{\mathrm{DB}}=\left(\gamma_{+}\right)_{\alpha \beta} P_{0}^{2}, \\
\left(Q_{\alpha}^{+(0)}, \bar{Q}_{\beta}^{-}\right)_{\mathrm{DB}}=-\left(\gamma_{\alpha} \gamma_{+} \gamma_{-}\right)_{\alpha \beta} P_{0}^{a},
\end{gathered}
$$

where we have used that the hamiltonian for the zero modes is the center-of-mass hamiltonian $H^{(0)}=\frac{1}{2} \boldsymbol{P}_{0}^{2}$. For the remainder of the supercharge $Q^{+}$, which does not contain the zero modes anymore (provided that the membrane coordinates are single-valued), the Dirac bracket reads

$$
\left(Q_{\alpha}^{+}, \bar{Q}_{\beta}^{+}\right)_{\mathrm{DB}}=\left(\gamma_{+}\right)_{\alpha \beta} \mathscr{M}^{2}-2\left(\gamma_{a} \gamma_{+}\right)_{\alpha \beta} \int \mathrm{d}^{2} \sigma w \varphi X^{a}+\cdots
$$

where the dots indicate the contribution from the surface terms. This relation plays a central role in the analysis of this paper.

So far we have been employing a $d=11$ notation for the spinors $\theta$. However, due to the gauge condition (2.16), the anticommuting coordinates are restricted to $\mathrm{SO}(9)$ spinors, satisfying

$$
\gamma_{1} \gamma_{2} \cdots \gamma_{9} \theta=\theta
$$

Furthermore we have

$$
\bar{\theta} \gamma_{-}=i \sqrt{2} \theta^{\dagger}, \quad \theta=\mathscr{C}^{-1} \boldsymbol{\theta}^{\dagger}
$$

where $\mathscr{C}$ is the $d=9$ charge conjugation matrix, which is symmetric and related to the $d=11$ charge conjugation matrix by $\mathscr{C}=-C \gamma_{11}$; we also note that the $\operatorname{SO}(9)$ gamma matrices satisfy $\gamma_{a}^{T}=\mathscr{C} \gamma_{a} \mathscr{C}^{-1}$. Henceforth we will choose $\mathscr{C}=\mathbf{1}$, so that the $\mathrm{SO}(9)$ gamma matrices are symmetric.

In subsequent sections we shall study the ground-state wave function of the supermembrane. For that purpose it is convenient to have an explicit representation for the operators associated with the fermionic coordinates. As a first step towards 
constructing such a representation we decompose the real $\mathrm{SO}(9)$ spinor coordinates $\theta$ into a single complex 8-component spinor $\lambda$, which transforms linearly under the $\mathrm{SO}(7) \times \mathrm{U}(1)$ subgroup of $\mathrm{SO}(9)$. This decomposition is effected by expressing the two eigenspinors of $\gamma_{9}$, defined by $\gamma_{9} \theta^{( \pm)}= \pm \theta^{( \pm)}$, into a complex $\operatorname{SO}(7)$ spinor $\lambda$, according to

$$
\theta^{(+)}=\frac{\lambda^{\dagger}+\lambda}{2^{5 / 4}}, \quad \theta^{(-)}=i \frac{\lambda^{\dagger}-\lambda}{2^{5 / 4}}
$$

The bosonic coordinates $X^{a}$ are then decomposed according to representations of this $\mathrm{SO}(7) \times \mathrm{U}(1)$ subgroup so we distinguish the components $X^{i}$ of an $\mathrm{SO}(7)$ vector $(i=1,2, \ldots, 7)$, while $X^{8}$ and $X^{9}$ are combined into a complex coordinate

$$
Z=\sqrt{\frac{1}{2}}\left(X^{8}+i X^{9}\right)
$$

which transforms under U(1). Similarly, the momenta are decomposed in terms of an $\mathrm{SO}(7)$ vector $P^{i}$ and a complex momentum $\mathscr{P}$ defined by

$$
\mathscr{P}=\sqrt{\frac{1}{2}}\left(P^{8}-i P^{9}\right)
$$

The normalization factors in (2.62)-(2.64) are chosen such that the nonvanishing Dirac brackets are equal to

$$
\begin{aligned}
& \left(X^{i}(\sigma), P^{j}\left(\sigma^{\prime}\right)\right)_{\mathrm{DB}}=\delta^{i j} \delta^{2}\left(\sigma-\sigma^{\prime}\right), \\
& \left(Z(\sigma), \mathscr{P}\left(\sigma^{\prime}\right)\right)_{\mathrm{DB}}=\delta^{2}\left(\sigma-\sigma^{\prime}\right), \\
& \left(\lambda_{\alpha}(\sigma), \lambda_{\beta}^{\dagger}\left(\sigma^{\prime}\right)\right)_{\mathrm{DB}}=-i w^{-1} \delta_{\alpha \beta} \delta^{2}\left(\sigma-\sigma^{\prime}\right) .
\end{aligned}
$$

The supercharges $Q_{\alpha}^{+}$can also be written as a complex $\operatorname{SO}(7)$ spinor. When expressed in terms of the above coordinates these charges take the form

$$
\begin{aligned}
& Q=\int \mathrm{d}^{2} \sigma\left[\left(P^{i} \Gamma_{i}+\frac{1}{2} w\left\{X^{i}, X^{j}\right\} \Gamma_{i j}-w\{Z, \bar{Z}\}\right) \lambda\right. \\
& \left.+\sqrt{2}\left(i \mathscr{P}+i w\left\{X^{i}, \bar{Z}\right\} \Gamma_{i}\right) \lambda^{\dagger}\right] \\
& Q^{\dagger}=\int \mathrm{d}^{2} \sigma\left[\left(-P^{i} \Gamma_{i}+\frac{1}{2} w\left\{X^{i}, X^{j}\right\} \Gamma_{i j}+w\{Z, \bar{Z}\}\right) \lambda^{\dagger}\right. \\
& \left.+\sqrt{2}\left(-i \overline{\mathscr{P}}+i w\left\{X^{i}, Z\right\} \Gamma_{i}\right) \lambda\right]
\end{aligned}
$$


where $\Gamma_{i}$ are the $\mathrm{SO}(7)$ gamma matrices ${ }^{\star}$. In the same notation the hamiltonian reads

$$
\begin{aligned}
H=\int \mathrm{d}^{2} \sigma & {\left[\frac{1}{2} w^{-1}\left(P^{i}\right)^{2}+w^{-1}|\mathscr{P}|^{2}\right.} \\
& +\frac{1}{4} w\left(\left\{X^{i}, X^{j}\right\}\right)^{2}+w\left|\left\{Z, X^{i}\right\}\right|^{2}+\frac{1}{2} w|\{Z, \bar{Z}\}|^{2} \\
& \left.+i w \lambda \Gamma_{i}\left\{X^{i}, \lambda^{\dagger}\right\}-\frac{1}{2} \sqrt{2} w \lambda\{Z, \lambda\}+\frac{1}{2} \sqrt{2} w \lambda^{\dagger}\left\{\bar{Z}, \lambda^{\dagger}\right\}\right] .
\end{aligned}
$$

The normalization of $Q$ and $Q^{\dagger}$ is such that

$$
\begin{aligned}
& \left(Q_{\alpha}, Q_{\beta}\right)_{\mathrm{DB}}=-\sqrt{2} \delta_{\alpha \beta} \int \mathrm{d}^{2} \sigma w \bar{Z} \varphi \\
& \left(Q_{\alpha}, Q_{\beta}^{\dagger}\right)_{\mathrm{DB}}=-2 i \delta_{\alpha \beta} H+2 i\left(\Gamma_{i}\right)_{\alpha \beta} \int \mathrm{d}^{2} \sigma w X^{i} \varphi
\end{aligned}
$$

\section{Area-preserving transformations and supersymmetric matrix models}

The analysis presented in the foregoing section has led us to the constraint (2.24) (or, (2.35)-(2.37) and (2.50-51)), which generates the group $\mathrm{G}$ of area-preserving diffeomorphisms. All physical quantities, such as the expression (2.33) for the membrane mass, must be invariant under this group, and this statement applies equally to the classical theory (where (2.24) constrains the space of solutions) and to the quantum theory (where (2.24) must be imposed as a constraint on the physical states). The group $\mathrm{G}$ and its associated Lie algebra play an important role in the following and are also of interest in their own right $[11,12]$. In this section, we summarize some properties of this group for spherical and toroidal membranes. Before going into the details we make some general remarks which also pertain to topologically more complicated membranes. We start by expanding the coordinates into a complete orthonormal basis of functions $Y^{A}(\sigma)$ on the membrane,

$$
\boldsymbol{X}(\sigma)=\sum_{A} \boldsymbol{X}^{A} Y_{A}(\sigma), \quad(A=0,1,2, \ldots)
$$

and likewise for the fermionic coordinates $\theta$ (or $\lambda$ ) and the momenta. The functions $Y_{A}$ may be chosen real, in which case there are no restrictions on the modes, or complex, in which case there are further restrictions from the reality of $\boldsymbol{X}$. The following notation allows us to discuss both options in a uniform manner. First we

* Our conventions are as follows: $\left\{\Gamma_{i}, \Gamma_{j}\right\}=2 \delta_{i j} \mathbf{1}, \Gamma_{i j} \equiv \frac{1}{2}\left[\Gamma_{i}, \Gamma_{j}\right], \Gamma_{i j k} \equiv \frac{1}{2}\left\{\Gamma_{i}, \Gamma_{j k}\right\}, \Gamma_{1} \ldots \Gamma_{7}=-i \mathbf{1}$. Also, $\Gamma_{i}=\Gamma_{i}^{\dagger}=-\Gamma_{i}^{\mathrm{T}}=-\Gamma_{i}^{*}$. 
define

$$
Y^{A}(\sigma) \equiv\left(Y_{A}(\sigma)\right)^{*}=\eta^{A B} Y_{B}(\sigma)
$$

where the matrix $\eta^{A B}$ satisfies $\eta^{A B} \eta_{B C}=\delta_{C}^{A}$ with $\eta_{A B} \equiv\left(\eta^{A B}\right)^{*}$. The normalization of the functions $Y_{A}$ is

$$
\int \mathrm{d}^{2} \sigma w(\sigma) Y^{A}(\sigma) Y_{B}(\sigma)=\delta_{B}^{A}
$$

or, equivalently,

$$
\int \mathrm{d}^{2} \sigma w(\sigma) Y_{A}(\sigma) Y_{B}(\sigma)=\eta_{A B}
$$

which shows that $\eta_{A B}$ is symmetric. The reality condition on the expansion coefficients of $\boldsymbol{X}(\boldsymbol{\sigma})$ then reads

$$
\boldsymbol{X}_{A} \equiv\left(\boldsymbol{X}^{A}\right)^{*}=\eta_{A B} \boldsymbol{X}^{B}
$$

Furthermore, completeness of the $Y_{A}$ implies

$$
\sum_{A} Y^{A}(\sigma) Y_{A}\left(\sigma^{\prime}\right)=\frac{1}{w(\sigma)} \delta^{2}\left(\sigma-\sigma^{\prime}\right)
$$

As explained in the previous section, area-preserving maps are expressed in terms of divergence-free vector fields, $\xi^{r}(\sigma)$; according to (2.39) these vector fields can be represented locally in terms of a scalar function $\xi(\sigma)$, which may or may not be globally defined. ${ }^{\star}$ We will concentrate on the subgroup of area-preserving maps generated by functions $\xi(\sigma)$ that are globally defined. As follows from (2.41), infinitesimal transformations can be expressed in terms of the Lie bracket defined in (2.40). Furthermore, the commutator of two infinitesimal transformations with parameters $\xi_{1}$ and $\xi_{2}$ yields an area-preserving transformation with parameter $\xi_{3}=\left\{\xi_{2}, \xi_{1}\right\}$. Therefore the structure constants of the area-preserving maps that are globally defined, are given in terms of the Lie bracket (2.40). In order to make this more explicit, we decompose the Lie bracket of $Y_{A}$ and $Y_{B}$ according to

$$
\left\{Y_{A}, Y_{B}\right\}=g_{A B}{ }^{C} Y_{C}=g_{A B C} Y^{C} \text {, }
$$

where indices of $g_{A B C}$ are raised and lowered by means of $\eta^{A B}$ and $\eta_{A B}$. Using the

* In the mathematics literature, the vector fields corresponding to functions $\xi(\sigma)$ that are globally defined, are called "hamiltonian vector fields"; if $\xi(\sigma)$ is not globally defined one speaks of "locally hamiltonian vector fields". See e.g. [19], p. 218. The latter contain harmonic vectors $\xi^{r}$ and homotopically nontrivial reparametrizations. 
normalization condition (3.3) it follows that $g_{A B C}$ is defined by

$$
\begin{aligned}
g_{A B C} & =\int \mathrm{d}^{2} \sigma w(\sigma) Y_{A}(\sigma)\left\{Y_{B}(\sigma), Y_{C}(\sigma)\right\} \\
& =\int \mathrm{d}^{2} \sigma \varepsilon^{r s} Y_{A}(\sigma) \partial_{r} Y_{B}(\sigma) \partial_{s} Y_{C}(\sigma) .
\end{aligned}
$$

Because the Lie bracket satisfies the Jacobi identity the structure constants will also satisfy this identity,

$$
\left.g_{[A B}{ }^{C}{ }_{g} D\right] C=0
$$

In the space of functions that are globally defined, it follows directly from the definition (3.7) that the structure constants $g_{A B C}$ are totally antisymmetric. As we will not consider compactified membranes, we will thus always be dealing with antisymmetric structure constants. Furthermore the zero-mode $Y_{0}(\sigma)=$ constant decouples from the other modes because

$$
g_{O B C}=g_{A O C}=g_{A B 0}=0 .
$$

It is now straightforward to substitute the expansion (3.1) and similar ones for the fermionic coordinates into the expressions derived in sect. 2. The lagrangian corresponding to (2.44) thus reads

$$
\begin{aligned}
L= & \frac{1}{2}\left(\partial_{0} X^{0}\right)^{2}+\frac{1}{2}\left|D_{0} X^{A}\right|^{2}+\bar{\theta}^{0} \gamma_{-} \partial_{0} \theta^{0}+\bar{\theta}_{A} \gamma_{-} D_{0} \theta^{A} \\
& -\frac{1}{4} g_{A B}{ }^{E} g_{C D E} X_{a}^{A} X_{b}^{B} X_{a}^{C} X_{b}^{D}-g_{A B C} X_{a}^{A} \bar{\theta}^{B} \gamma_{-} \gamma^{a} \theta^{C}
\end{aligned}
$$

where we have separately written the zero modes (corresponding to $A=0$ ) and the nonzero modes with indices $A, B, \ldots$ ranging from 1 to $\infty$. The covariant derivatives in (3.10) are defined by

$$
D_{0} X_{a}^{A}=\partial_{0} X_{a}^{A}-g_{B C}{ }^{A} \omega^{B} X_{a}^{C}, \quad D_{0} \theta^{A}=\partial_{0} \theta^{A}-g_{B C}{ }^{A} \omega^{B} \theta^{C},
$$

where $\omega^{A}$ is the gauge field associated with time-dependent area-preserving transformations. The lagrangian (3.10) is invariant under time-dependent transformations, whose infinitesimal form is given by

$$
\delta X_{a}^{A}=g_{B C}{ }^{A} \xi^{B} X_{a}^{C}, \quad \delta \theta^{A}=g_{B C}{ }^{A} \xi^{B} \theta^{C}, \quad \delta \omega^{A}=D_{0} \xi^{A}
$$

so that the zero modes are invariant by virtue of (3.9) and the nonzero modes transform in the adjoint representation. 
As in sect. 2, the hamiltonian associated with (3.10) in the gauge $\omega^{\mathcal{A}}=0$ leads to an equation for the membrane mass $\mathscr{M}$,

$$
\mathscr{M}^{2}=\boldsymbol{P}_{A} \cdot \boldsymbol{P}^{A}+\frac{1}{2} g_{A B}{ }^{E} g_{C D E} X_{a}^{A} X_{b}^{B} X_{a}^{C} X_{b}^{D}+2 g_{A B C} X_{a}^{A} \bar{\theta}^{B} \gamma_{-} \gamma^{a} \theta^{C}
$$

which does not contain the zero modes. The relevant supercharge is the part of $Q^{+}$, defined in (2.54), that pertains to the nonzero modes,

$$
Q=\left(2 P_{a}^{A} \gamma^{a}+g_{B C}^{A} X_{b}^{B} X_{c}^{C} \gamma^{b c}\right) \theta_{A}
$$

As shown in (2.59) the Dirac bracket of $Q$ with itself yields (3.13) and the constraint $\varphi$, whose components are

$$
\varphi_{A}=g_{A B C}\left(\boldsymbol{P}^{B} \cdot \boldsymbol{X}^{C}+\ddot{\theta}^{B} \gamma_{-} \theta^{C}\right) \approx 0
$$

The theory defined by (3.13)-(3.15) contains an infinite number of degrees of freedom. In order to make it well-defined, one would like to have some kind of regularization. This can be achieved by restricting the indices $A, B, C, \ldots$ to a finite range between 1 and some finite number $\Lambda$. The original theory would then be obtained in the limit $\Lambda \rightarrow \infty$. In general, this limiting procedure may destroy some of the symmetries of the theory, and it is not clear which of these will be restored in this limit. The most severe of these problems are cured if one can replace the full group $\mathrm{G}$ of area-preserving transformations by a finite-dimensional symmetry group $\mathrm{G}_{A}$, which in the limit $\Lambda \rightarrow \infty$ coincides with $\mathrm{G}$. The structure constant $g^{A B C}$ can then be replaced by the structure constants $f^{A B C}$ of the finite-dimensional group $\mathrm{G}_{\Lambda}$, which satisfy

$$
\lim _{A \rightarrow \infty} f^{A B C}=g^{A B C}
$$

The existence of such a group $G_{\Lambda}$ guarantees that supersymmetry is not affected, as this symmetry rests upon the existence of a Jacobi identity for the structure constants (it also depends on the space-time dimension through the condition (2.5)). The application of this regularization thus leads us to a class of $N=16$ supersymmetric matrix models with hamiltonian

$$
H=\operatorname{Tr}\left(\frac{1}{2} \boldsymbol{P}^{2}+\frac{1}{4}\left[X_{a}, X_{b}\right]^{2}+\left[X_{a}, \bar{\theta}\right] \gamma_{-} \gamma^{a} \theta\right)
$$

where $\boldsymbol{P}, \boldsymbol{X}$ and $\theta$ are matrices that take their values in the Lie algebra of $\mathrm{G}$. Surprisingly enough, the quantum-mechanical version of these models coincides with the models proposed sometime ago in [15] ${ }^{\star}$. However, it is not guaranteed that the group $G_{A}$ will always exist. This has been demonstrated only for spherical membranes $[11,12]$. In that case $\mathrm{G}_{\Lambda}$ is equal to the group $\operatorname{SU}(N)$, where $N$ and $\Lambda$

^ These models are reductions of supersymmetric Yang-Mills theories to $1+0$ dimensions. The field $\omega$ introduced in sect. 2 corresponds to the timelike component of the gauge fields. 
are related by $\Lambda=N^{2}-1$. Of course, subtle questions about the precise meaning of the limit $\Lambda \rightarrow \infty$ still remain and will require further study. However, we shall ignore such questions here and turn to a more detailed discussion of the area-preserving transformations for the sphere and the torus.

\subsection{AREA-PRESERVING MAPS ON THE SPHERE}

On the sphere one conventionally takes the spherical harmonics $Y_{I m}(\theta, \varphi)$ as basis functions, where we exclude the zero mode, so that the integers $l$ and $m$ satisfy $l \geq 1, \quad|m| \leq l$. With this basis we have $w(\theta, \varphi)=(4 \pi)^{-1} \sin \theta$. We choose the Condon-Shortley phase convention for the $Y_{l m}$ (we follow the definitions of [20], except for the normalization of the $Y_{l m}$ which differs by a factor $\sqrt{4 \pi}$ ),

$$
\left(Y_{l}^{m}\right)^{*}=(-)^{m} Y_{l}^{-m},
$$

so that

$$
\eta_{(l m)\left(l^{\prime} m^{\prime}\right)}=(-)^{m} \delta_{l-l^{\prime}} \delta_{m+m^{\prime}},
$$

where $\delta_{l}$ denotes the Kronecker symbol $\delta_{l 0}$. The Lie bracket of two spherical harmonics then reads

$$
\begin{aligned}
\left\{Y_{l_{1} m_{1}}, Y_{l_{2} m_{2}}\right\} & =\frac{4 \pi}{\sin \theta}\left(\frac{\partial Y_{l_{1} m_{1}}}{\partial \theta} \frac{\partial Y_{l_{2} m_{2}}}{\partial \varphi}-\frac{\partial Y_{l_{1} m_{1}}}{\partial \varphi} \frac{\partial Y_{l_{2} m_{2}}}{\partial \theta}\right) \\
& =g_{l_{1} m_{1}, l_{2} m_{2}}{ }^{l_{3} m_{3}} Y_{l_{3} m_{2}} .
\end{aligned}
$$

It should be obvious that $g_{l_{1} m_{1}, l_{2} m_{2}, l_{3} m_{3}}=0$, unless $m_{1}+m_{2}+m_{3}=0$. Furthermore, one can verify that $l_{1}+l_{2}+l_{3}$ must be odd, for instance, by comparing the parity of both sides of (3.20), and that $l_{3} \leq l_{1}+l_{2}-1$. Using the antisymmetry of the structure constants it then follows that the structure constants only differ from zero if

$$
\left|l_{1}-l_{2}\right|+1 \leq l_{3} \leq l_{1}+l_{2}-1, \quad m_{1}+m_{2}+m_{3}=0 \text {. }
$$

Another way to see this is by writing the spherical harmonics as symmetric traceless homogeneous polynomials of three cartesian coordinates $x_{1}, x_{2}, x_{3}$ :

$$
Y_{l m}(\theta, \varphi)=r^{-l} a_{i_{1} \ldots i_{i}}^{(l m)} x_{i_{1}} \ldots x_{i_{l}}, \quad\left(r^{2}=x_{1}^{2}+x_{2}^{2}+x_{3}^{2}\right)
$$

in which case the Lie bracket takes the form

$$
\{A, B\}=4 \pi r \varepsilon_{i j k} x_{i} \partial_{j} A \partial_{k} B .
$$


Substitution of (3.22) into (3.23) leads to the same restrictions on $l_{1}, l_{2}$ and $l_{3}$ as listed in (3.21). The representation (3.23) also shows that the structure constants for $l_{1}=l_{2}=l_{3}=1$ are proportional to those of $\mathrm{SO}(3)$.

In [12] it was shown that $g_{l_{1} m_{1}, l_{2} m_{2}, l_{3} m_{3}}$ are the $N \rightarrow \infty$ limit of $\mathrm{SU}(N)$ structure constants. Let us first indicate how $\mathrm{SU}(N)$ emerges in the truncation of the spherical harmonics to a finite set. This truncation is effected by restricting $l$ to $l \leq N-1$, which leaves us with precisely $N^{2}-1$ functions $Y_{l m}$. To each $Y_{l m}$, which corresponds to the symmetric traceless homogeneous polynomials (3.22), we can generally assign an $N$-dimensional matrix by constructing the corresponding symmetric traceless product of $\mathrm{SO}(3)$ generators $L_{i}$ in the $N$-dimensional representation $\left(\operatorname{spin} s=\frac{1}{2}(N-1)\right)$,

$$
Y_{l m} \rightarrow T_{l m}=4 \pi\left(\frac{N^{2}-1}{4}\right)^{(1-l) / 2} a_{i_{1} \ldots i_{l}}^{(l m)} L_{i_{1}} \ldots L_{i_{l}} .
$$

As is well-known, the $L_{i}$ satisfy the equations

$$
\left[L_{i}, L_{j}\right]=i \varepsilon_{i j k} L_{k}, \quad L_{i}^{\dagger}=L_{i}, \quad L^{2}=\frac{N^{2}-1}{4} \mathbf{1},
$$

as well as the pseudo-reality condition

$$
L_{i}^{*}=L_{i}^{\mathrm{T}}=-\omega L_{i} \omega^{-1}
$$

The matrices (2.24) are traceless by virtue of the tracelessness of the tensors $a^{(l m)}$. The dimension of the representation is chosen such that the $T_{I m}$ with $l \leq N-1$ form a complete set of traceless $N \times N$ matrices. This can easily be seen by writing them as the traceless part of $L_{ \pm}^{p} L_{3}^{q}$, with $L_{ \pm}$the familiar raising and lowering operators, which are clearly independent, provided $p+q \leq N-1$. Using the symmetry property (3.26), it then follows that the $T_{l m}$ with even (odd) $l \geq N$ can be expanded as a linear combination of the $T_{l m}$ with even (odd) $l \leq N-1$. Note that the hermiticity of the $T_{l m}$ follows from the phase convention adopted for the spherical harmonics, so that

$$
\left(T_{l}^{m}\right)^{\dagger}=(-)^{m} T_{l}^{-m}
$$

From their completeness property it is obvious that the $T_{l m}$ are the generators of $\mathrm{SU}(N)$ in the defining representation, and we obtain the structure constants from

$$
\left[T_{l_{1} m_{1}}, T_{l_{2} m_{2}}\right]=i f_{l_{1} m_{1}, l_{2} m_{2}}{ }^{l_{3} m_{3}} T_{l_{3} m_{3}} \quad\left(\left|m_{i}\right| \leq l_{i} \leq N-1\right) .
$$

Just as the structure constants of the area-preserving transformations, the $\mathrm{SU}(N)$ structure constants $f_{l_{1} m_{1}, l_{2} m_{2}, l_{3} m_{3}}$ are only different from zero if $l_{1}+l_{2}+l_{3}$ is odd 
(this follows from applying (3.26) to both sides of (3.28)), $l_{3} \leq l_{1}+l_{2}-1$ and $m_{1}+m_{2}+m_{3}=0$. Therefore we have the same restrictions on $l_{i}$ and $m_{i}$ as given in (3.21), except that one should keep in mind that, in the case of $\operatorname{SU}(N)$, there is the additional restriction that $l_{i} \leq N-1$.

Due to (3.24) $f_{l_{1} m_{1}, l_{2} m_{2}, l_{3} m_{3}}$ will converge to $g_{l_{1} m_{1}, l_{2} m_{2}, l_{3} m_{3}}$ as $N \rightarrow \infty$ [12]. Eq. (3.24) also implies that the $T_{l m}$ transform as tensor operators under rotations and once this is known the $\mathrm{SU}(N)$ structure constants defined by (3.28) are determined by group theory $[21,12]$ up to the calculation of the reduced matrix elements. One gets (without loss of generality, we have assumed that $l_{1} \leq l_{2} \leq l_{3}$ while $l_{1}+l_{2}-l_{3}$ is an odd positive integer)

$$
\begin{aligned}
f_{l_{1} m_{1}, l_{2} m_{2}, l_{3} m_{3}=} & -4 \pi i\left(\prod_{i=1}^{3} \sqrt{2 l_{i}+1}\right)\left(\begin{array}{ccc}
l_{1} & l_{2} & l_{3} \\
m_{1} & m_{2} & m_{3}
\end{array}\right) \\
& \times\left\{\begin{array}{ccc}
l_{1} & l_{2} & l_{3} \\
s & s & s
\end{array}\right\}(-) \frac{{ }^{N} R_{N}\left(l_{1}\right) R_{N}\left(l_{2}\right)}{R_{N}\left(l_{3}\right)},
\end{aligned}
$$

where $\left(\begin{array}{lll}l_{1} & l_{2} & l_{3} \\ m_{1} & m_{2} & m_{3}\end{array}\right)$ and $\left\{\begin{array}{lll}l_{1} & l_{2} & l_{3} \\ s & s & s\end{array}\right\}$ are the $3 j$-symbol and the $6 j$-symbol, respectively [20], with $s=\frac{1}{2}(N-1)$, while the function $R_{N}$ is defined by

$$
R_{N}(l)=\sqrt{\frac{(N+l) !\left(N^{2}-1\right)^{1-l}}{(N-l-1) !}} .
$$

In the large- $N$ limit, the expression to the right of the $3 j$-symbol converges to

$$
\begin{aligned}
\left\{\begin{array}{ccc}
l_{1} & l_{2} & l_{3} \\
s & s & s
\end{array}\right\}(-)^{N} & \frac{R_{N}\left(l_{1}\right) R_{N}\left(l_{2}\right)}{R_{N}\left(l_{3}\right)} \stackrel{N \rightarrow \infty}{\longrightarrow}\left(1+l_{1}+l_{2}+l_{3}\right) l_{1} ! l_{2} ! l_{3} !(-)^{l_{3}-1} \\
& \times \sqrt{\frac{\left(l_{1}+l_{2}-l_{3}\right) !\left(l_{1}+l_{3}-l_{2}\right) !\left(l_{2}+l_{3}-l_{1}\right) !}{\left(1+l_{1}+l_{2}+l_{3}\right) !}} \sum_{n=0}^{l_{1}+l_{2}-l_{3}} \frac{n(-)^{n}}{F(n)}
\end{aligned}
$$

where

$$
F(n)=n !\left(l_{1}+l_{2}-l_{3}-n\right) !\left(l_{1}-n\right) !\left(l_{2}-n\right) !\left(n+l_{3}-l_{1}\right) !\left(n+l_{3}-l_{2}\right) ! .
$$

The large- $N$ limit of (3.29) coincides with the structure constants $g_{l_{1} m_{1}, l_{2} m_{2}, l_{3} m_{3}}$ for the full group of area-preserving transformations. The mathematics underlying this result $[12,22]$ is quite intriguing, and could lead to the possibility of approximating other infinite-dimensional Lie algebras of symplectic diffeomorphisms on homogeneous manifolds by large- $N$ matrix algebras. 


\subsection{AREA-PRESERVING MAPS ON THE TORUS}

Choosing torus coordinates $0 \leq \phi_{1}, \phi_{2}<2 \pi$ the basis functions $Y_{A}$ are labelled by two-dimensional vectors $\boldsymbol{m}=\left(m_{1}, m_{2}\right)$ with $m_{1}, m_{2}$ integer numbers. They are defined by

$$
Y_{m}(\phi)=\mathrm{e}^{i m \cdot \phi}
$$

where $\boldsymbol{m} \cdot \boldsymbol{\phi}=m_{1} \phi_{1}+m_{2} \phi_{2}$. Again we will exclude the zero mode, so that $\boldsymbol{m} \neq 0$. Furthermore we have $w(\phi)=\left(4 \pi^{2}\right)^{-1}$, and $\eta_{m n}=\delta_{m+n}$. For the Lie bracket of $Y_{m}$ and $Y_{n}$, one easily finds

$$
\left\{Y_{m}, Y_{n}\right\}=-4 \pi^{2}(m \times n) Y_{m+n}
$$

where $\boldsymbol{m} \times \boldsymbol{n} \equiv m_{1} n_{2}-m_{2} n_{1}$. The structure constants $g_{A B C}$ follow directly from (3.7) and read

$$
g_{m n k}=-4 \pi^{2}(m \times n) \delta_{m+n+k}
$$

The elements of the Lie algebra associated with $\mathrm{G}$ are thus labelled by the set of nonzero two-dimensional vectors $\boldsymbol{m}$ with integer coordinates. The commutator of two generators corresponding to two vectors of this lattice is then equal to the generator corresponding to the sum of the two vectors, multiplied by $i$ times the oriented area of the parallelogram enclosed by the two vectors. Generators associated with parallel vectors thus commute. There exits an infinite variety of Cartan subalgebras, each infinite dimensional, consisting of the generators corresponding to the set of parallel vectors $\boldsymbol{m}=\lambda \boldsymbol{n}$, with $\boldsymbol{n}$ fixed and $\lambda$ all nonzero integers.

The algebra corresponding to the structure constant (3.35) has been discussed in connection with the theory of incompressible fluids in [19]. Recently, it was emphasized that it contains subalgebras that are isomorphic to the Virasoro algebra [23]. One such subalgebra was explicitly given; its generators take the form

$$
L_{m}=\frac{1}{4 \pi^{2}} \sum_{k \neq 0} \frac{1}{k} Y_{(k, m+k)}
$$

More generally, solutions are obtained by taking a (logarithmically diverging) sum of the $Y_{m}$ along a straight line in the 2-dimensional plane. For instance, one may take

$$
L_{m}=\frac{1}{4 \pi^{2}} \sum_{k \neq 0} \frac{1}{k p} Y_{(m, k p)}, \quad \text { or } \quad L_{m}=\frac{1}{4 \pi^{2}} \sum_{k \neq 0} \frac{1}{k p} Y_{(k p, m+k)}
$$

where $p$ is some nonzero integer. However, some caution is required with the infinite sums in (3.36)-(3.37), as the formal expressions for $L_{m}$ do not correspond to 
differentiable functions of the torus coordinates $\phi_{1}$ and $\phi_{2}$. The Lie algebra based on (3.35) allows for a nontrivial central extension,

$$
\left\{Y_{m}, Y_{n}\right\}=-4 \pi^{2}(\boldsymbol{m} \times \boldsymbol{n}) Y_{m+n}+\boldsymbol{c} \cdot \boldsymbol{m} \delta_{m+n},
$$

where $\boldsymbol{c}$ is a real two-dimensional vector. This result was also noted in [23]. Furthermore, one can enlarge the torus algebra to include fermionic generators $X_{r}$ with (anti)commutation relations ${ }^{\star}$ (to avoid confusion with the usual symbol for the anticommutator, we replace $-\left(1 / 4 \pi^{2}\right)\{$,$\left.\} by [],\right)$

$$
\begin{aligned}
{\left[Y_{m}, Y_{n}\right] } & =(\boldsymbol{m} \times \boldsymbol{n}) Y_{m+n}, \\
\left\{X_{r}, X_{s}\right\} & =Y_{r+s}, \\
{\left[Y_{m}, X_{r}\right] } & =(\boldsymbol{m} \times \boldsymbol{r}) X_{m+r},
\end{aligned}
$$

where the fermionic generators $X_{r}$ are labelled by the set of two-dimensional vectors $\boldsymbol{r}=\left(r_{1}, r_{2}\right)$, with $r_{1}$ and $r_{2}$ ranging either over the integers, or half integers (so that we get four different algebras, two of which are isomorphic to each other).

\section{The supermembrane as a supersymmetric quantum-mechanical model}

In this section we combine the previous results and study the properties of the supermembrane ground state. So far, we have not been able to prove or disprove the assertion that the supermembrane has massless states, although most of our results indicate that the ground state is massive. However, we stress that more work is needed before one can reach a definitive conclusion regarding this issue, and we hope that the results described here will pave the way for a more rigorous treatment of supermembranes which goes beyond perturbative (semi-classical) arguments.

The quantization of the supermembrane is straightforward in the $\operatorname{SO}(7) \times U(1)$ formulation that we have presented in sect. 2. The coordinates are therefore $X^{i}(\sigma)$, $Z(\sigma), \bar{Z}(\sigma)$ and $\lambda_{\alpha}(\sigma)$, with corresponding canonical momenta $P^{i}(\sigma), \mathscr{P}(\sigma), \overline{\mathscr{P}}(\sigma)$ and $\lambda_{\alpha}^{\dagger}(\sigma)$. The (anti)commutators of the operators associated with the coordinates and the momenta are given by the Dirac brackets (2.65) multiplied with an extra factor $i$. The operators $P^{i}, \mathscr{P}, \overline{\mathscr{P}}$ and $\lambda^{\dagger}$ can then be realized on wave functions (or rather functionals) $\Psi\left[X^{i}, Z, \bar{Z}, \lambda\right]$ by

$$
\begin{array}{ll}
P^{i}(\sigma)=-i \frac{\partial}{\partial X^{i}(\sigma)}, & \mathscr{P}(\sigma)=-i \frac{\partial}{\partial Z(\sigma)}, \\
\overline{\mathscr{P}}(\sigma)=-i \frac{\partial}{\partial \bar{Z}(\sigma)}, & \lambda^{\dagger}(\sigma)=\frac{1}{w} \frac{\partial}{\partial \lambda(\sigma)} .
\end{array}
$$

* This superextension of the algebra has been obtained in collaboration with Garreis (see [24]) and $J$. Wess. 
It is now straightforward to write the relevant formulae from sect. 2 in this representation. Before doing so, we "regularize" the supermembrane theory by decomposing the coordinates and the momenta in terms of a finite set of function $Y^{0}(\sigma)$ and $Y^{A}(\sigma)$ with $A=1, \ldots, \Lambda$. As explained in sect. 3, the structure constants $g_{A B C}$ of the group of area-preserving transformations are then replaced by the structure constants $f_{A B C}$ of a finite compact Lie group $\mathrm{G}$, with dimension

$$
\operatorname{dim} \mathrm{G}=\Lambda
$$

In the limit $\Lambda \rightarrow \infty$ the group $\mathrm{G}$ is assumed to coincide with the group of area-preserving transformations. This procedure turns the supermembrane into a model of supersymmetric quantum mechanics [13,14] and leads precisely to the supersymmetric matrix models that have been constructed in [15]. An important consequence of this approach is that supersymmetry remains preserved, while the invariance under area-preserving maps is approximated by the invariance under $\mathbf{G}$. For membranes topologically equivalent to $S^{2}$ the group $\mathrm{G}$ is equal to $\operatorname{SU}(N)$ and the limit $N \rightarrow \infty$ has been shown to yield the full group of area-preserving transformations [12]. However, in this section the precise nature of $G$ does not play an important role.

The model that we will be considering in this section is thus based on a finite set of coordinates $X_{i}^{A}, Z^{A}, \bar{Z}^{A}$ and $\lambda_{\alpha}^{A}$, together with their canonically conjugate momenta $P_{i}^{A}, \mathscr{P}^{A}, \overline{\mathscr{P}}^{A}$ and $\lambda_{\alpha}^{A \dagger}$. Here, the index $A$ labels the adjoint representation of $\mathrm{G}$. There are also the zero-mode (or center-of-mass) coordinates $X_{i}^{0}, Z^{0}, \bar{Z}^{0}$ and $\lambda_{\alpha}^{0}$, but as we have already emphasized, these decouple entirely from the other coordinates, and do not contribute to the mass of the supermembrane states. The (anti)commutation relations corresponding to (2.65) are

$$
\begin{aligned}
& {\left[X_{i}^{A}, P_{j B}\right]=i \delta_{i j} \delta_{B}^{A},} \\
& {\left[Z^{A}, \mathscr{P}_{B}\right]=\left[\bar{Z}^{A}, \overline{\mathscr{P}}_{B}\right]=i \delta_{B}^{A},} \\
& \left\{\lambda_{\alpha}^{A}, \lambda_{\beta B}^{\dagger}\right\}=\delta_{\alpha \beta} \delta_{B}^{A},
\end{aligned}
$$

while all other (anti)commutators vanish. The conjugate momenta can thus be represented by the operators

$$
\begin{array}{ll}
P_{i A}=-i \frac{\partial}{\partial X_{i}^{A}}, & \mathscr{P}_{A}=-i \frac{\partial}{\partial Z^{A}} \\
\overline{\mathscr{P}}_{A}=-i \frac{\partial}{\partial \bar{Z}^{A}}, & \lambda_{\alpha A}^{\dagger}=\frac{\partial}{\partial \lambda_{\alpha}^{A}},
\end{array}
$$


in agreement with (4.1), and the states of the theory correspond to the wave functions $\Psi\left(X_{i}^{A}, Z^{A}, \bar{Z}^{A}, \lambda_{\alpha}^{A}\right)$. The latter are elements of the Grassmann algebra generated by $\lambda_{\alpha}^{A}$ and may be expressed as ${ }^{\star}$

$$
\Psi=\sum_{k=0}^{8 A} \Phi_{A_{1} \ldots A_{k}}^{\alpha_{1} \ldots \alpha_{k}}(X, Z, \bar{Z}) \lambda_{\alpha_{1}}^{A_{1}} \lambda_{\alpha_{2}}^{A_{2}} \ldots \lambda_{\alpha_{k}}^{A_{k}} .
$$

The norm of the state $\Psi$ can then be defined through

$$
\|\Psi\|^{2}=\sum_{k=0}^{8 \Lambda} \frac{1}{k !}\left\|\Phi_{A_{1} \ldots A_{k}}^{\alpha_{1} \ldots \alpha_{k}}\right\|^{2},
$$

with the usual $L^{2}$-norms for the coefficient functions $\Phi_{A_{1} \ldots A_{k}}^{\alpha_{1} \ldots \alpha_{k}}$. Of course, one also has the customary distinction between bosonic and fermionic states according to whether only even or odd powers of $\lambda_{\alpha}^{A}$ appear.

We next make the appropriate substitutions in the supercharge operators of sect. 2. The supercharges that pertain to the nonzero modes, follow directly from the $\mathrm{SO}(7) \times \mathrm{U}(1)$ covariant expressions in (2.66) and take the form

$$
\begin{aligned}
Q_{\alpha}= & \left\{-i \Gamma_{\alpha \beta}^{i} \frac{\partial}{\partial X_{i}^{A}}+\frac{1}{2} f_{A B C} X_{i}^{B} X_{j}^{C} \Gamma_{\alpha \beta}^{i j}-f_{A B C} Z^{B} \bar{Z}^{C} \delta_{\alpha \beta}\right\} \lambda_{\beta}^{A} \\
& +\sqrt{2}\left\{\delta_{\alpha \beta} \frac{\partial}{\partial Z^{A}}+i f_{A B C} X_{i}^{B} \overline{Z^{C}} \Gamma_{\alpha \beta}^{i}\right\} \frac{\partial}{\partial \lambda_{\beta A}}, \\
Q_{\alpha}^{\dagger}= & \left\{i \Gamma_{\alpha \beta}^{i} \frac{\partial}{\partial X_{i}^{A}}+\frac{1}{2} f_{A B C} X_{i}^{B} X_{j}^{C} \Gamma_{\alpha \beta}^{i j}+f_{A B C} Z^{B} \bar{Z}^{C} \delta_{\alpha \beta}\right\} \frac{\partial}{\partial \lambda_{\beta A}} \\
& +\sqrt{2}\left\{-\delta_{\alpha \beta} \frac{\partial}{\partial \bar{Z}^{A}}+i f_{A B C} X_{i}^{B} Z^{C} \Gamma_{\alpha \beta}^{i}\right\} \lambda_{\beta}^{A} .
\end{aligned}
$$

These charges define a supersymmetric quantum-mechanical model, whose hamiltonian follows from the $\left\{Q, Q^{\dagger}\right\}$ anticommutator. In order to exhibit this, let us evaluate the anticommutators of the supercharge operators $Q$ and $Q^{\dagger}$. After a somewhat lengthy calculation, using the antisymmetry of $f^{A B C}$ as well as the Jacobi

^ Observe that we suppress the dependence on the zero-mode coordinates in (4.5). We will return to this shortly. 
identities, one arrives at the following superalgebra

$$
\begin{aligned}
& \left\{Q_{\alpha}, Q_{\beta}\right\}=2 \sqrt{2} \delta_{\alpha \beta} \bar{Z}^{A} \varphi_{A}, \\
& \left\{Q_{\alpha}^{\dagger}, Q_{\beta}^{\dagger}\right\}=2 \sqrt{2} \delta_{\alpha \beta} Z^{A} \varphi_{A}, \\
& \left\{Q_{\alpha}, Q_{\beta}^{\dagger}\right\}=2 \delta_{\alpha \beta} H-2 i \Gamma_{\alpha \beta}^{i} X_{i}^{A} \varphi_{A} .
\end{aligned}
$$

This result is consistent with the Dirac brackets (2.65), with the operators $H$ and $\varphi_{A}$ corresponding to the contribution from the nonzero modes to the hamiltonian (2.67) and the constraint (2.51). The explicit expression for this hamiltonian, which is directly related to the membrane mass $\mathscr{M}$, reads

$$
H=\frac{1}{2} \mathscr{M}^{2}=H_{\mathrm{b}}+H_{\mathrm{f}},
$$

where

$$
H_{\mathrm{b}}=-\frac{1}{2} \frac{\partial^{2}}{\partial X_{A}^{i} \partial X_{i}^{A}}-\frac{\partial^{2}}{\partial Z_{A} \partial \bar{Z}^{A}}+V(X, Z, \bar{Z})
$$

with positive potential $V$ given by

$$
V(X, Z, \bar{Z})=\frac{1}{4} f_{A B}^{E} f_{C D E}\left\{X_{i}^{A} X_{j}^{B} X_{i}^{C} X_{j}^{D}+4 X_{i}^{A} Z^{B} X_{i}^{C} \bar{Z}^{D}+2 Z^{A} \bar{Z}^{B} \bar{Z}^{C} Z^{D}\right\},
$$

and

$$
H_{\mathrm{f}}=i f_{A B C} X_{i}^{A} \lambda_{\alpha}^{B} \Gamma_{\alpha \beta}^{i} \frac{\partial}{\partial \lambda_{\beta C}}+\frac{1}{2} \sqrt{2} f_{A B C}\left(Z^{A} \lambda_{\alpha}^{B} \lambda_{\alpha}^{C}-\bar{Z}^{A} \frac{\partial}{\partial \lambda_{\alpha B}} \frac{\partial}{\partial \lambda_{\alpha C}}\right)
$$

The algebra (4.8) still contains the operators $\varphi^{A}$, which are the components of the constraint (2.51), and given by

$$
\varphi^{A}=f^{A B C}\left(X_{i B} \frac{\partial}{\partial X_{i}^{C}}+Z_{B} \frac{\partial}{\partial Z^{C}}+\bar{Z}_{B} \frac{\partial}{\partial \bar{Z}^{C}}+\lambda_{\alpha B} \frac{\partial}{\partial \lambda_{\alpha}^{C}}\right) .
$$

Obviously, $\varphi^{A}$ are just the generators of the group $G$, which must vanish on physical states, i.e.,

$$
\varphi^{A} \Psi=0
$$

Consequently the wave functions corresponding to physical states must be invariant under $\mathrm{G}$ (or the full group of area-preserving diffeomorphisms). On physical states one thus recovers the usual supersymmetry algebra. The expressions (4.9)-(4.14) 
precisely coincide with the results of [15], where quantum-mechanical models were discussed with up to 16 supercharges. Hence we have established that the supermembrane is a limiting case of this class of models.

The zero modes, which are not contained in the quantum-mechanical models of [15], lead also to corresponding supercharges, as we have already discussed in sect. 2. In the $\mathrm{SO}(7) \times \mathrm{U}(1)$ notation, there is one complex charge associated with $Q^{-}$ and one with $Q^{+}$(cf. (2.54), where we denote the latter by $Q^{+(0)}$ to indicate that it contains only contributions from the zero modes. In the representation (4.4) these charges read

$$
\begin{aligned}
Q_{\alpha}^{-} & =\lambda_{\alpha}^{0}, \quad Q_{\alpha}^{-\dagger}=\frac{\partial}{\partial \lambda_{\alpha}^{0}}, \\
Q_{\alpha}^{(0)} & =-i \Gamma_{\alpha \beta}^{i} \frac{\partial}{\partial X_{i}^{0}} \lambda_{\beta}^{0}+\sqrt{2} \frac{\partial}{\partial Z^{0}} \frac{\partial}{\partial \lambda_{\alpha}^{0}}, \\
Q_{\alpha}^{(0) \dagger} & =i \Gamma_{\alpha \beta}^{i} \frac{\partial}{\partial X_{i}^{0}} \frac{\partial}{\partial \lambda_{\beta}^{0}}+\sqrt{2} \frac{\partial}{\partial \bar{Z}^{0}} \lambda_{\alpha}^{0} .
\end{aligned}
$$

It is easy to determine the supersymmetry algebra for the above charges, which is the quantum-mechanical analogue of $(2.58)$ in $\mathrm{SO}(7) \times \mathrm{U}(1)$ notation. This algebra contains the hamiltonian

$$
H^{(0)}=-\frac{1}{2} \frac{\partial^{2}}{\partial X_{i}^{0} \partial X_{i}^{0}}-\frac{\partial^{2}}{\partial Z^{0} \partial \bar{Z}^{0}},
$$

which is just the transverse kinetic energy of the membrane. The wave function associated with the zero modes is simply a plane-wave solution in terms of the transverse coordinates $X_{i}^{0}, Z^{0}$ and $\bar{Z}^{0}$ with a certain transverse momentum, multiplied by an arbitrary function of the fermionic zero modes $\lambda^{0}$. This wave function thus describes 128 bosonic states $1, \lambda_{\alpha}^{0} \lambda_{\beta}^{0}, \ldots$ and 128 fermionic states $\lambda_{\alpha}^{0}, \lambda_{\alpha}^{0} \lambda_{\beta}^{0} \lambda_{\gamma}^{0}, \ldots$. Under $\mathrm{SO}(9)$, these transform as the $44 \oplus 84$ and 128 representations. The $128+128$ independent wave functions transform under the supercharge operators (4.15) as the states of a massless $d=11$ supergravity multiplet. To see this, it is convenient to choose a Lorentz frame in which the transverse momentum vanishes, so that the charge $Q^{(0)}$ vanishes and one is only left with $Q^{-}$. Consequently, if the wave function (4.5) associated with the ground state of the nonzeromode system is not degenerate, then the supermembrane ground state constitutes precisely a massless supermultiplet.

According to the above arguments, the zero modes are no longer relevant, and we have to determine the nature of the ground state corresponding to the hamiltonian $H$ which governs the nonzero modes. According to (4.9), massless states $\Psi$ must 
obey the Schrödinger equation

$$
H \Psi=0 .
$$

From the supersymmetry algebra, it follows that $H$ can be written as

$$
H=\frac{1}{16}\left\{Q_{\alpha}, Q_{\alpha}^{\dagger}\right\}
$$

The hamiltonian $H$ is thus a positive operator, which vanishes if and only if the ground-state wave function $\Psi$ is a singlet under supersymmetry, in which case

$$
Q_{\alpha} \Psi=Q_{\alpha}^{\dagger} \Psi=0
$$

Although this condition ensures that the ground state is massless, it does not immediately imply that the ground state constitutes the desired supermultiplet. In $d=11$ dimensions one has to require separately that $\Psi$ is also a singlet under $\mathrm{SO}(9){ }^{\star}$ For future purposes let us list the $\mathrm{SO}(9)$ generators in terms of the coordinates and momenta introduced above. It is convenient to decompose them into "orbital" and "spin" parts according to

$$
J^{a b}=L^{a b}+S^{a b},
$$

where

$$
\begin{aligned}
& L_{i j}=X_{i}^{A} \frac{\partial}{\partial X_{j}^{A}}-X_{j}^{A} \frac{\partial}{\partial X_{i}^{A}}, \\
& L_{89}=i Z^{A} \frac{\partial}{\partial Z^{A}}-i \bar{Z}^{A} \frac{\partial}{\partial \bar{Z}^{A}}, \\
& L_{i+}=X_{i}^{A} \frac{\partial}{\partial Z^{A}}-\bar{Z}^{A} \frac{\partial}{\partial X_{i}^{A}}, \\
& L_{i-}=X_{i}^{A} \frac{\partial}{\partial \bar{Z}^{A}}-Z^{A} \frac{\partial}{\partial X_{i}^{A}},
\end{aligned}
$$

and

$$
\begin{aligned}
& S_{i j}=\frac{1}{2} \lambda_{\alpha}^{A} \Gamma_{\alpha \beta}^{i j} \frac{\partial}{\partial \lambda_{\beta}^{A}}, \quad S_{89}=-\frac{1}{2} i \lambda_{\alpha}^{A} \frac{\partial}{\partial \lambda_{\alpha}^{A}}+i c_{0}, \\
& S_{i-}=\frac{i}{2 \sqrt{2}} \frac{\partial}{\partial \lambda_{\alpha}^{A}} \Gamma_{\alpha \beta}^{i} \frac{\partial}{\partial \lambda_{\beta A}}, \quad S_{i+}=\frac{i}{2 \sqrt{2}} \lambda_{\alpha}^{A} \Gamma_{\alpha \beta}^{i} \lambda_{\beta A} .
\end{aligned}
$$

\footnotetext{
${ }^{\star}$ In lower-dimensional space-times $\Psi$ must transform nontrivially under the $\operatorname{SO}(d-2)$ group of transverse rotations in order that the ground-state constitutes a supergravity multiplet.
} 
Note the appearance of the "normal-ordering" constant $c_{0} \equiv 2 \Lambda$ in $S_{89}$. There is an associated hermitean $\mathrm{U}(1)$ charge operator $J_{+-}$which reads

$$
J_{+-} \equiv i J_{89}=\bar{Z}^{A} \frac{\partial}{\partial \bar{Z}^{A}}-Z^{A} \frac{\partial}{\partial Z^{A}}+\frac{1}{2} \lambda_{\alpha}^{A} \frac{\partial}{\partial \lambda_{\alpha}^{A}}-c_{0},
$$

(with corresponding definitions for $S_{+-}$and $L_{+-}$). Defining the charge $q$ of any operator $\mathcal{O}$ by means of $\left[J_{+-}, \mathcal{O}\right]=q \mathcal{O}$, we see the variables $X_{i}^{A}, Z^{A}, \bar{Z}^{A}$ and $\lambda_{\alpha}^{A}$ carry the $U(1)$ charges $0,-1,+1$ and $\frac{1}{2}$, respectively.

Our main task is now to solve (4.17), or equivalently (4.19), for some G-invariant wave function $\Psi$. We expect that the method of solving (4.17) for finite $\Lambda$ cannot be used for purely bosonic membranes, because the ground-state energy of the bosonic membrane will diverge in the limit $\Lambda \rightarrow \infty$ and needs to be renormalized (see, e.g. [25]). Since this is a nonrenormalizable theory there is an inherent ambiguity in the calculation of the finite part of the infinite renormalization. On the other hand, if one succeeds in finding a state obeying (4.19) for the supermembrane, this state will remain a proper ground state in the limit $\Lambda \rightarrow \infty$. Nevertheless, we cannot a priori exclude the possibility that the lowest eigenvalue of $H$ is strictly positive for finite $A$ but only tends to zero as $\Lambda \rightarrow \infty$. At any rate, we expect that the Bose-Fermi symmetry leads to the usual softening of divergences associated with the large- $\Lambda$ limit.

Up to this point, the analysis is completely analogous to the corresponding one for superstrings (a detailed discussion may be found in [26], sect. 11.7). The much more difficult part of the problem, however, resides in the nonzero mode part of $\Psi$. First of all, the hamiltonian (4.9) describes an interacting theory and not a free theory as in superstring theory. Secondly, the constraint (4.14) has no analog in string theory. There, one only demands invariance of the physical Hilbert space under rigid (i.e., length-preserving) translations that are generated by the operator $N_{\mathrm{L}}-N_{\mathrm{R}}$, which does not mix different oscillator modes. The group of area-preserving diffeomorphisms is much larger and, in particular, does not admit an invariant split into positively and negatively indexed modes.

In order to facilitate the calculations, one can make the additional assumption that $\Psi$ is an $\operatorname{SO}(9)$ singlet. As alluded to above, this is in fact necessary if one wants to recover $d=11$ supergravity as a "low-energy limit" from the supermembrane.

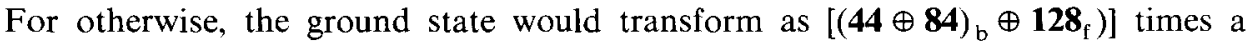
nonsinglet representation of $\mathrm{SO}(9)$ and would therefore describe states other than those of the $d=11$ supergravity multiplet. Unfortunately, the requirement of $\operatorname{SO}(9)$ invariance does not lead to significant simplifications, so that this approach is not particularly useful. We refer the reader to the appendix for a more detailed analysis of the structure of $\mathrm{SO}(9)$-invariant wave functions. However, one can show that the ground-state wave function cannot factorize into a bosonic and a fermionic function, i.e., it cannot be of the form $\Psi=\Psi_{b} \otimes \Psi_{f}$, with either $\Psi_{b}$ or $\Psi_{f}$ (or both) SO(9) 
or $\mathrm{G}$ invariant. The reason is that $H_{\mathrm{f}}$, defined in (4.12), can be written as a product of two operators, a bosonic one equal to the bosonic coordinates, and a fermionic one, bilinear in the fermion operators, which both transform as a vector under $\mathrm{SO}(9)$ and in the adjoint representation of $\mathrm{G}$. Sandwiching $H_{\mathrm{f}}$ between the groundstate wave functions, it follows from the $\mathrm{SO}(9)$ or $\mathrm{G}$ invariance of either $\Psi_{\mathrm{b}}$ or $\Psi_{\mathrm{f}}$ that $\left(\Psi, H_{\mathrm{f}} \Psi\right)$ must vanish. Therefore, as a result of $(4.17),\left(\Psi, H_{\mathrm{b}} \Psi\right)=\left(\Psi_{\mathrm{b}}, H_{\mathrm{b}} \Psi_{\mathrm{b}}\right)$ $=0$. Because $H_{\mathrm{b}}$ is a positive operator, this implies that $\Psi_{\mathrm{b}}$ must vanish. This situation is in sharp contrast to superstrings where the (nonzero mode) ground-state factorizes into a bosonic and a fermionic $\mathrm{SO}(8)$ singlet, and where one has a mode-by-mode cancellation of the vacuum energies.

In general, the relevant equations $Q \Psi=Q^{\dagger} \Psi=0$ are very difficult to solve. Therefore we will now consider two special cases to illustrate some of the difficulties. The first one is a truncation of the membrane theory, in which we discard the coordinates $Z^{A}, \bar{Z}^{A}$ and $\lambda_{8}^{A}$. We accordingly split the $\operatorname{SO}(7)$ spinor indices $\alpha, \beta, \ldots$ into $i, j, \ldots=1, \ldots, 7$ and $\alpha, \beta, \ldots=8$ and make use of the fact that (see, e.g. $[27,28])$

$$
\left(\Gamma^{i}\right)_{j 8}=-i \delta_{j}^{i}, \quad\left(\Gamma^{i}\right)_{j k}=i c_{i j k},
$$

where $c_{i j k}$ are the octonionic structure constants obeying

$$
c_{i j m} c^{k l m}=2 \delta_{i j}^{k l}-\frac{1}{6} \varepsilon_{i j k l m n p} c^{m n p}
$$

as well as a number of other relations which can be found in [28]. In this truncation the supercharges (4.7) take the form

$$
\begin{aligned}
& Q_{8}=\left\{\frac{\partial}{\partial X_{i}^{A}}+\frac{1}{2} c^{i j k} f_{A B C} X_{j}^{B} X_{k}^{C}\right\} \lambda_{i}^{A}, \\
& Q_{8}^{\dagger}=\left\{-\frac{\partial}{\partial X_{i}^{A}}+\frac{1}{2} c^{i j k} f_{A B C} X_{j}^{B} X_{k}^{C}\right\} \frac{\partial}{\partial \lambda_{A}^{i}} .
\end{aligned}
$$

The symmetry of this theory is now reduced to $N=1$ supersymmetry, the $\mathrm{G}_{2}$ subgroup of $\operatorname{SO}(9)$ and $\mathrm{G}$. The equation $Q \Psi=Q^{\dagger} \Psi=0$ can easily be solved and one finds two $\mathrm{G}_{2} \times \mathrm{G}$ invariant solutions,

$$
\begin{aligned}
& \Psi_{1}=\left(\prod_{i, A} \lambda_{i}^{A}\right) \exp \left\{\frac{1}{6} c^{i j k} f_{A B C} X_{i}^{A} X_{j}^{B} X_{k}^{C}\right\}, \\
& \Psi_{2}=\exp \left\{-\frac{1}{6} c^{i j k} f_{A B C} X_{i}^{A} X_{j}^{B} X_{k}^{C}\right\} .
\end{aligned}
$$


It is amusing that in the membrane limit these two solutions become

$$
\begin{aligned}
& \Psi_{1}[X(\sigma), \lambda(\sigma)]=\left(\prod_{i, \sigma} \lambda_{i}(\sigma)\right) \exp \left\{\frac{1}{6} \int \mathrm{d}^{2} \sigma \varepsilon^{r s} c_{i j k} X^{i} \partial_{r} X^{j} \partial_{s} X^{k}\right\}, \\
& \Psi_{2}[X(\sigma), \lambda(\sigma)]=\exp \left\{-\frac{1}{6} \int \mathrm{d}^{2} \sigma \varepsilon^{r s} c_{i j k} X^{i} \partial_{r} X^{j} \partial_{s} X^{k}\right\},
\end{aligned}
$$

so that the ground-state wave functionals are exponentials of a Wess-Zumino-Witten term, with corresponding torsion proportional to $c_{i j k}$. However, both solutions (4.27) fail to be square-integrable, and this problem persists for (4.28). Thus, there is no supersymmetric ground state, so that this truncation has no massless states. From the analogy with ordinary $N=1$ supersymmetric quantum mechanics, this is what one would have intuitively expected for the full supermembrane, too, as the differential operator, which appears in (4.7), is $\pm \partial / \partial X+X^{2}$, rather than $\pm \partial / \partial X$ $+X$ as in superstring theory [14]. However, the argument is vitiated by (amongst other things) the nonexistence of an $\mathrm{SO}(9)$-invariant (or even $\mathrm{SO}(7)$-invariant) three-index tensor analogous to $c_{i j k}$. Observe also that both solutions in (4.27) are singlets in their bosonic and fermionic factors. This does not contradict our findings above, because the wave functions do not tend to zero at spatial infinity, and for such functions the hamiltonian $H_{b}$ is not a positive operator.

The second truncation which we will consider, consists in discarding the variables $X_{i}^{A}$ and $\lambda_{i}^{A}$, thus retaining only $Z^{A}, \bar{Z}^{A}$ and $\lambda^{A} \equiv \lambda_{8}^{A}$. This corresponds to a membrane moving in a $d=4$ dimensional space-time. The supercharges follow directly from (4.7) and read

$$
\begin{gathered}
Q=\sqrt{2} \frac{\partial}{\partial Z^{A}} \frac{\partial}{\partial \lambda_{A}}-f_{A B C} Z^{A} \bar{Z}^{B} \lambda^{C} \\
Q^{\dagger}=-\sqrt{2} \frac{\partial}{\partial \bar{Z}^{A}} \lambda^{A}+f_{A B C} Z^{A} \bar{Z}^{B} \frac{\partial}{\partial \lambda_{C}} .
\end{gathered}
$$

It is clear that the ground state cannot factorize into a bosonic and fermionic part and therefore we proceed from the ansat ${ }^{\star}$

$$
\Psi=\Phi_{0}(Z, \bar{Z})+\sum_{k \geq 1} \Phi_{A_{1} \ldots A_{2 k}}(Z, \bar{Z}) \lambda^{A_{1}} \ldots \lambda^{A_{2 k}}
$$

where the coefficient functions $\Phi^{A_{1} \ldots A_{2 k}}$ are completely antisymmetric in the indices $A_{1}, \ldots, A_{2 k}$. To make life as simple as possible, we take $\mathrm{G}$ equal to $\mathrm{SU}(2)$, so that

\footnotetext{
"We could also choose $\Psi$ such that only odd powers of $\lambda$ appear.
} 
$A, B, C, \ldots=1,2,3$, and $f^{A B C}=\varepsilon^{A B C}$. The decomposition (4.30) then simplifies to

$$
\Psi=\varphi_{0}(Z, \bar{Z})+\varepsilon^{A B C} \varphi^{A}(Z, \bar{Z}) \lambda^{B} \lambda^{C} .
$$

(We choose a real basis for the adjoint representation of SU(2), so the position of indices is immaterial).

Requiring $Q \Psi=Q^{\dagger} \Psi=0$, we get

$$
\varepsilon^{A B C} Z^{A} \bar{Z}^{B} \varphi^{C}(Z, \bar{Z})=0
$$

which tells us that

$$
\varphi^{A}=Z^{A} \varphi_{1}+\bar{Z}^{A} \varphi_{2}
$$

and three more equations,

$$
\begin{gathered}
Z^{A} \frac{\partial \varphi_{1}}{\partial \bar{Z}^{A}}+3 \varphi_{2}+\bar{Z}^{A} \frac{\partial \varphi_{2}}{\partial \bar{Z}^{A}}=0 \\
2 \sqrt{2} \varepsilon^{A B C}\left\{Z^{B} \frac{\partial \varphi_{1}}{\partial Z^{C}}+\bar{Z}^{B} \frac{\partial \varphi_{2}}{\partial Z^{C}}\right\}=\varepsilon^{A B C} Z^{B} \bar{Z}^{C} \varphi_{0} \\
\sqrt{2} \frac{\partial \varphi_{0}}{\partial \bar{Z}^{A}}=2\left[(Z \cdot \bar{Z}) Z^{A}-Z^{2} \bar{Z}^{A}\right] \varphi_{1}+2\left[\bar{Z}^{2} Z^{A}-(Z \cdot \bar{Z}) Z^{A}\right] \varphi_{2} .
\end{gathered}
$$

Upon multiplication by $Z^{A}$ and $\bar{Z}^{A}$, (4.36) leads to

$$
\begin{aligned}
& \varphi_{1}=\frac{1}{\sqrt{2}} \frac{1}{(Z \cdot \bar{Z})^{2}-Z^{2} \bar{Z}^{2}} \bar{Z}^{A} \frac{\partial \varphi_{0}}{\partial \bar{Z}^{A}}, \\
& \varphi_{2}=-\frac{1}{\sqrt{2}} \frac{1}{(Z \cdot \bar{Z})^{2}-Z^{2} \bar{Z}^{2}} Z^{A} \frac{\partial \varphi_{0}}{\partial \bar{Z}^{A}} .
\end{aligned}
$$

Substituting this result back into the previous equations, it turns out that (4.34) is identically satisfied, while (4.35) and (4.36) lead to

$$
\begin{gathered}
\varepsilon^{A B C} Z^{A} \bar{Z}^{B} \frac{\partial \varphi_{0}}{\partial Z^{C}}=\varepsilon^{A B C} Z^{A} \bar{Z}^{B} \frac{\partial \varphi_{0}}{\partial \bar{Z}^{C}}=0 \\
\tilde{H} \varphi_{0} \equiv\left\{H_{\mathrm{b}}+\frac{\varepsilon^{A B C} Z^{B} \bar{Z}^{C}}{(Z \cdot \bar{Z})^{2}-Z^{2} \bar{Z}^{2}} \varepsilon^{A D E}\left(\bar{Z}^{D} \frac{\partial}{\partial \bar{Z}^{E}}-Z^{D} \frac{\partial}{\partial Z^{E}}\right)\right\} \varphi_{0}=0 .
\end{gathered}
$$


Here $H_{\mathrm{b}}$ is the hamiltonian defined in (4.10), which in this case reads

$$
H_{\mathrm{b}}=-\frac{\partial}{\partial Z^{A}} \frac{\partial}{\partial \bar{Z}^{A}}+\frac{1}{2}\left[(Z \cdot \bar{Z})^{2}-Z^{2} \bar{Z}^{2}\right]
$$

According to the constraint equations (4.14), the wave function must be $\mathrm{SU}(2)$ invariant, in which case eqs. (4.38) are obviously satisfied. Hence we are left with a Schrödinger equation for an SU(2)-invariant wave function $\varphi_{0}$, given by (4.39). The corresponding hamiltonian, $\tilde{H}$, consists of a linear combination of $H_{\mathrm{b}}$, which is the hamiltonian for a bosonic membrane, and an extra term.

For the class of wave functions for which the hamiltonian is self-adjoint, we find that

$$
\left(\varphi_{0}, H_{\mathrm{b}} \varphi_{0}\right)=\int \mathrm{d}^{3} Z \mathrm{~d}^{3} \bar{Z}\left\{\left|\frac{\partial \varphi_{0}}{\partial Z^{A}}\right|^{2}+\frac{1}{2}\left[(Z \cdot \bar{Z})^{2}-Z^{2} \bar{Z}^{2}\right]\left|\varphi_{0}\right|^{2}\right\}
$$

which is positive because

$$
(Z \cdot \bar{Z})^{2}-Z^{2} \bar{Z}^{2} \geq 0
$$

Under the same conditions, we have

$$
\left(\varphi_{0},\left(\tilde{H}-H_{\mathrm{b}}\right) \varphi_{0}\right)=\int \mathrm{d}^{3} Z \mathrm{~d}^{3} \bar{Z} \frac{1}{2} \frac{\varepsilon^{A B C} Z^{B} \bar{Z}^{C}}{(Z \cdot \bar{Z})^{2}-Z^{2} \bar{Z}^{2}} \varepsilon^{A D E}\left(\bar{Z}^{D} \frac{\partial}{\partial \bar{Z}^{E}}-Z^{D} \frac{\partial}{\partial Z^{E}}\right)\left|\varphi_{0}\right|^{2}
$$

Because

$$
\varepsilon^{A D E}\left(\bar{Z}^{D} \frac{\partial}{\partial \bar{Z}^{E}}-Z^{D} \frac{\partial}{\partial Z^{E}}\right) \frac{\varepsilon^{A B C} Z^{B} \bar{Z}^{C}}{(Z \cdot \bar{Z})^{2}-Z^{2} \bar{Z}^{2}}=0
$$

the integrand in (4.43) can be written as a total divergence, which suggests that one can rewrite (4.43) as a surface integral. However, one has to take into account that the integrand has a singularity whenever $(Z \cdot \bar{Z})^{2}=Z^{2} \bar{Z}^{2}$. This happens when $Z^{A}$ becomes proportional to a real vector (or, in other words, whenever the two vectors $\operatorname{Re} Z^{A}$ and $\operatorname{Im} Z^{A}$ are aligned). Therefore, the integral (4.43) splits into two terms, one corresponding to the surface integral associated with large distances $(Z \cdot \bar{Z} \rightarrow$ $\infty$ ), which yields a positive contribution, and another one corresponding to the contribution from the singularities, which turns out to be negative. To show this more explicitly, on may choose a parametrization in terms of the $\mathrm{SU}(2)$-invariant 
variables

$$
\zeta \equiv Z^{A} Z^{A}, \quad \bar{\zeta} \equiv \bar{Z}^{A} \bar{Z}^{A}, \quad \xi \equiv \sqrt{(Z \cdot \bar{Z})^{2}-Z^{2} \bar{Z}^{2}}
$$

It is not hard to see that $\tilde{H}-H_{\mathrm{b}}$ is now equal to

$$
\tilde{H}-H_{\mathrm{b}}=\frac{2}{\xi} \sqrt{\xi^{2}+|\xi|^{2}} \frac{\partial}{\partial \xi} .
$$

Furthermore, on SU(2)-invariant functions we have

$$
\mathrm{d}^{3} Z \mathrm{~d}^{3} \bar{Z} \propto \frac{\xi \mathrm{d} \xi \mathrm{d} \zeta \mathrm{d} \bar{\zeta}}{\sqrt{\xi^{2}+|\zeta|^{2}}}
$$

up to "angular" variables whose integral yields an irrelevant (positive) constant $c$. Substituting (4.46)-(4.47) into (4.43), and performing the integral over $\xi$, we then find

$$
\left(\varphi_{0},\left(\tilde{H}-H_{\mathrm{b}}\right) \varphi_{0}\right)=-c \int \mathrm{d} \zeta \mathrm{d} \bar{\zeta}\left|\varphi_{0}(\xi=0, \zeta, \bar{\zeta})\right|^{2}
$$

where we have dropped the contribution at $\xi=\infty$, which is proportional to $\left|\varphi_{0}\right|^{2}$ at spatial infinity. Therefore we have shown that for wave functions vanishing at infinity, the energy of a supermembrane will be lower than that of a corresponding bosonic membrane.

On the other hand, imposing the boundary condition that $\varphi_{0}$ vanishes when $Z \cdot \bar{Z} \rightarrow \infty$, one can see that no solution of (4.39) exists, as $\bar{H}$ is an elliptic differential operator (see e.g. [29], p. 320 ff.). Consequently, solutions that are subject to these boundary conditions do not have zero energy. We should emphasize, however, that the above boundary condition is not implied by square-integrability ${ }^{\star}$, and we have not been able to establish the existence or nonexistence of a general square-integrable solution to (4.39).

It is now evident that the general case with arbitrary $N$ is even harder to tackle because the number of coefficient functions in (4.30) as well as the number of SU( $N)$ invariant variables analogous to (4.45) is further increased as $N$ becomes larger. In particular, there seems no real advantage anymore to replacing the

* This is, for instance, demonstrated by the function $f(\xi, \zeta, \bar{\zeta})=\zeta \exp \left[-\frac{1}{2} \xi^{1 / 4}|\zeta|^{2}-\frac{1}{2} \xi^{2}\right]$. which does not satisfy the above boundary condition, as $\lim _{|\xi| \rightarrow \infty} f(0, \zeta, \bar{\zeta})=\infty$. but nevertheless $\int_{0}^{\infty} \mathrm{d} \xi \int \mathrm{d}^{2} \xi|f(\xi, \zeta, \bar{\zeta})|^{2}<\infty$ ! 
second-order equation (4.17) by the first-order equation (4.19), since decoupling these equations will automatically lead to higher-order equations.

\section{Note added}

After this paper was completed we learnt that Claudson and Halpern (see [15]) consider wave functions similar to (4.27). Furthermore, we have meanwhile calculated the Witten index for the SU(2) model discussed at the end of sect. 4 along the lines of ref. [31] and found that it vanishes. This is consistent with the conclusion that there are no massless states.

\section{Appendix}

\section{STRUCTURE OF SO(9)-INVARIANT WAVE FUNCTIONS}

We here briefly describe how to construct $\mathrm{SO}(9)$-invariant wave functions which do not factorize into bosonic and fermionic parts that are separately $\mathrm{SO}(9)$ invariant. The basic idea is to first consider nontrivial SO(9) representations in either sector and then fold them together to form a singlet. This is completely obvious for the $\mathrm{SO}(7)$ subgroup of $\mathrm{SO}(9)$ and the nontrivial part of the analysis involves the generators $J_{i \pm}$ which are nonlinearly realized on the Grassmann algebra, cf. (4.22). As is well-known, any $\mathrm{SO}(9)$ representation can be characterized by its highest weight or, equivalently, by its Dynkin label (see e.g. [30]). In the present case this label consists of four positive integers $\left(a_{1} a_{2} a_{3} a_{4}\right)$, the first three of which indicate the $\mathrm{SO}(7)$ representation and the last of which is associated with the U(1) charge operators $L_{+-}$and $S_{+-}$. The highest-weight state $\left|\left(a_{1} a_{2} a_{3} a_{4}\right)\right\rangle$ must be annihilated by the raising operators $L_{i+}$ and $S_{i+}$, i.e.

$$
L_{i+}\left|\left(a_{1} a_{2} a_{3} a_{4}\right)\right\rangle_{\mathrm{b}}=0, \quad \text { or } \quad S_{i+}\left|\left(a_{1} a_{2} a_{3} a_{4}\right)\right\rangle_{\mathrm{f}}=0 \text {, }
$$

for a bosonic or fermionic representation, respectively. Of course, it must also be annihilated by the remaining raising operators of the $S O(7)$ subgroup but this (and analogous statements) will be understood in the following. The representation is then generated by applying the lowering operators $L_{i-}$ for the bosonic representations, or $S_{i-}$ for the fermionic representations, until one reaches the lowest-weight state; in this procedure, the U(1) charge $a_{4}$ is changed by one unit at each step. From the discussion in sect. 4 we learn that the fermionic wave functions have a maximum $U(1)$ charge which is equal to the normal-ordering constant $c_{0}=2 \Lambda$, so we will restrict ourselves to representations with $\left|a_{4}\right| \leq c_{0}$.

We will now illustrate how this works by looking at various examples, first in the bosonic sector. So let us start with

$$
\left|\left(000 c_{0}\right)\right\rangle_{\mathrm{b}}=\bar{Z}^{A_{1}} \ldots \bar{Z}^{A_{c_{0}}} \text {. }
$$

Obviously this state transforms under the symmetric tensor representation of the group $\mathrm{G}$ which is associated with the indices $A_{1}, \ldots, A_{c_{0}}$, but because $\mathrm{G}$ commutes 
with $\mathrm{SO}(9)$, this aspect is not very important. Clearly, the state (A.2) is an $\operatorname{SO}(7)$ singlet and annihilated by $L_{i+}$ (use the explicit expressions in (4.21)). Acting on it with $L_{i-}$, we obtain

$$
L_{i-}\left(\bar{Z}^{A_{1}} \ldots \bar{Z}^{A_{c_{0}}}\right)=c_{0} X_{i}^{\left(A_{1}\right.} \bar{Z}^{A_{2}} \ldots \bar{Z} \bar{Z}_{\left.c_{0}\right)} .
$$

The U(1) charge of (A.3) is $\left(c_{0}-1\right)$ while the G-representation content is evidently unaltered. Continuing in this fashion, we get

$$
\begin{aligned}
& L_{i-} L_{j-}\left(\bar{Z}^{A_{1}} \ldots \bar{Z}^{A_{c_{0}}}\right) \\
& \quad=c_{0}\left(c_{0}-1\right) X_{i}^{\left(A_{1}\right.} X_{j}^{A_{2}} \bar{Z}^{A_{3}} \ldots \bar{Z}^{\left.A_{c_{0}}\right)}-c_{0} \delta_{i j} Z^{\left(A_{1}\right.} \bar{Z}^{A_{2}} \bar{Z}^{A_{3}} \ldots \bar{Z}^{\left.A_{c_{0}}\right)},
\end{aligned}
$$

and so on. Hence, we just obtain a generalization of the usual $\mathrm{SO}(9)$ spherical harmonics. To also have an example with $a_{4}=c_{0}-1$, one may start from any of the following states

$$
\begin{aligned}
\left|\left(* * * c_{0}-1\right)\right\rangle_{\mathrm{b}}= & X_{i}^{\left[B_{1}\right.} \bar{Z}^{\left.B_{2}\right]} \bar{Z}^{A_{2}} \ldots \bar{Z}^{A_{r}}, \\
& X_{i}^{\left[B_{1}\right.} X_{j}^{B_{2}} \bar{Z}^{\left.B_{3}\right]} \bar{Z}^{A_{2}} \ldots \bar{Z}^{A_{r}}, \quad \text { or } \\
& X_{i}^{\left[B_{1}\right.} X_{j}^{B_{2}} X_{k}^{B_{3}} \bar{Z}^{\left.B_{4}\right]} \bar{Z}^{A_{2}} \ldots \bar{Z}^{A_{r}},
\end{aligned}
$$

where $(* * *)$ is the appropriate $\operatorname{SO}(7)$ label. Owing to the antisymmetry in the indices $B_{1}, B_{2}, \ldots$ the states (A.5) are anihilated by $L_{i+}$.

The construction in the fermionic sector is similar. Since, by (4.23), the highestweight state contains the maximal number of $\lambda$ 's, it is more convenient to start with the lowest-weight state. The analogue of (A.2) is then

$$
\left|\left(000-c_{0}\right)\right\rangle_{\mathrm{f}}=1,
$$

which is annihilated by $S_{i-}$. The action of $S_{i+}$ now produces the state

$$
S_{i+}\left|\left(000-c_{0}\right)\right\rangle_{\mathrm{f}}=\frac{i}{2 \sqrt{2}} \lambda^{A} \Gamma^{i} \lambda_{A},
$$

which has charge $-c_{0}+1$. The analogue of (A.5) is the set of states

$$
\lambda^{B_{1}} \lambda^{B_{2}}, \quad \lambda^{B_{1}} \Gamma^{i} \lambda^{B_{2}}-\frac{2}{c_{0}} \delta^{B_{1} B_{2}} \lambda^{C} \Gamma^{i} \lambda_{C}, \quad \lambda^{B_{1}} \Gamma^{i j} \lambda^{B_{2}}, \quad \lambda^{B_{1}} \Gamma^{i j k} \lambda^{B_{2}} .
$$

An $\mathrm{SO}(9)$ singlet can now be formed by folding together the same bosonic and fermionic $\mathrm{SO}(9)$ representations. The resulting wavefunction can then be turned into a singlet with respect to $G$ by contraction with an appropriate bosonic function of 
$\mathrm{SO}(9)$ singlet variables such as $X_{i}^{A} X_{i}^{B}+Z^{A} \bar{Z}^{B}+Z^{B} \bar{Z}^{A}$, etc. For instance, from (A.2) and (A.6), we can construct the following $\mathrm{SO}(9)$-singlet wavefunction

$$
\begin{aligned}
\Psi= & \left|\left(000 c_{0}\right)\right\rangle_{\mathrm{b}} \otimes\left|\left(000-c_{0}\right)\right\rangle_{\mathrm{f}} \\
& +\alpha L_{i-}\left|\left(000 c_{0}\right)\right\rangle_{\mathrm{b}} \otimes S_{i+}\left|\left(000-c_{0}\right)\right\rangle_{\mathrm{f}} \\
& +\beta L_{i-} L_{j-}\left|\left(000 c_{0}\right)\right\rangle_{\mathrm{b}} \otimes S_{i+} S_{j+}\left|\left(000-c_{0}\right)\right\rangle_{\mathrm{f}} \\
& +\gamma L_{i-} L_{i-}\left|\left(000 c_{0}\right)\right\rangle_{\mathrm{b}} \otimes S_{j+} S_{j+}\left|\left(000-c_{0}\right)\right\rangle_{\mathrm{f}} \\
& +\cdots
\end{aligned}
$$

The coefficients $\alpha, \beta, \gamma, \ldots$ are determined from the requirement $J_{i \pm} \Psi=0$. Using the $\mathrm{SO}(9)$ commutation relations and the known $\mathrm{U}(1)$ charges together with $L_{i j}\left|\left(000 c_{0}\right)\right\rangle_{\mathrm{b}}=S_{i j}\left|\left(000-c_{0}\right)\right\rangle_{\mathrm{f}}=0$ we find

$$
\alpha=\frac{1}{c_{0}}, \quad \beta=\frac{1}{2 c_{0}\left(c_{0}-1\right)}, \quad \gamma=\frac{\beta}{2 c_{0}+5} .
$$

After contraction with an appropriate bosonic wavefunction, (A.9) can also be expressed as

$$
\begin{aligned}
\Psi= & \Phi_{A_{1} \ldots A_{c_{0}}}(X, Z, \bar{Z}) \\
& \times\left\{\bar{Z}^{A_{1}} \ldots \bar{Z}^{A_{c_{0}}}+\frac{i}{2 \sqrt{2}} \lambda^{B} \Gamma^{j} \lambda_{B} X_{j}^{A_{1}} \bar{Z}^{A_{2}} \ldots \bar{Z}^{A_{c_{0}}}+\cdots\right\}
\end{aligned}
$$

Another example is

$$
\begin{aligned}
\Psi^{\prime}= & \Phi_{B_{1} B_{2} A_{2} \ldots A_{c_{0}}}(X, Z, \bar{Z}) \\
& \times\left\{\lambda^{B_{1}} \lambda^{B_{2}} \bar{Z}^{A_{2}} \ldots \bar{Z}^{A_{c_{0}}}+\frac{i}{2 \sqrt{2}} \lambda^{B_{1}} \lambda^{B_{2}} \lambda^{C} \Gamma^{j} \lambda_{C} X_{j}^{A_{2}} \bar{Z}^{A_{3}} \ldots \bar{Z}^{A_{c_{0}}}+\cdots\right\} .
\end{aligned}
$$

It is not difficult to verify directly that indeed $J_{i \pm}=L_{i \pm}+S_{i \pm}$ vanish on $\Psi$ and $\Psi^{\prime}$, at least to the order given. Obviously, there is a multitude of possibilities and very little hope of a complete classification. One can also prove that the supermembrane wave function for a massless ground-state cannot just be of the form (A.11). This follows directly from the observation that $H_{\mathrm{f}} \Psi$ contains no $\lambda$-independent term for $\Psi$ given by (A.11), so that $H_{\mathrm{b}} \Psi$ must vanish up to order $\lambda^{2}$ for a massless 
ground state. From the fact that $H_{\mathrm{b}}$ is positive, it then follows that $\Psi$ must in fact vanish. This conclusion is already suggested by the fact that (A.11) is an eigenfunction of both $\boldsymbol{L}^{2}$ and $\boldsymbol{S}^{2}$, while the hamiltonian does not commute with these operators. A bothersome feature is that the degree of the $\mathrm{SO}(9)$ "spherical harmonic" is larger than or equal to $c_{0}=2 \Lambda$ and therefore increases without bound as $\Lambda \rightarrow \infty$. It is hard to see what reasonably behaved wavefunction could ensure square-integrability of $\Psi, \Psi^{\prime}, \ldots$ or any linear combination thereof in this limit.

\section{References}

[1] E. Bergshoeff, E. Sezgin and P.K. Townsend, Phys. Lett. 189B (1987) 75; Ann. of Phys. 185 (1988) 330

[2] L. Brink and J.H. Schwarz, Phys. Lett. 100B (1981) 310; W. Siegel, Phys. Lett. 128B (1983) 397; Class. Quantum Grav. 2 (1985) L95--L97

[3] M.B. Green and J.H. Schwarz, Phys. Lett. 136B (1984) 367; Nucl. Phys. B243 (1984) 285

[4] J. Hughes, J. Liu and J. Polchinsky, Phys. Lett. 180B (1986) 370

[5] M. Henneaux and L. Mezincescu, Phys. Lett. 152B (1985) 340

[6] M.J. Duff, T. Inami, C.N. Pope, E. Sezgin and K.S. Stelle, Nucl. Phys. B297 (1988) 515

[7] L. Mezincescu, R. Nepomechie and P. van Nieuwenhuizen, preprint ITP-SB-87-43

[8] L. Brink and H.B. Nielsen, Phys. Lett. 45B (1973) 332

[9] K. Kikkawa and M. Yamasaki, Progr. Theor. Phys. 76 (1986) 1379

[10] I. Bars, C.N. Pope and E. Sezgin, Phys. Lett. 198B (1987) 455; I. Bars, preprint USC-87/HEP06 (1987)

[11] J. Goldstone, unpublished

[12] J. Hoppe, MIT Ph.D. Thesis, 1982, and in Proc. Int. Workshop on Constraint's theory and relativistic dynamics, ed. G. Longhi and L. Lusanna (World Scientific, 1987)

[13] H. Nicolai, J. Phys. A9 (1976) 1497, A10 (1977) 2143

[14] E. Witten, Nucl. Phys. B188 (1981) 513

P. Salomonson and J.W. van Holten, Nucl. Phys. B196 (1982) 509

M. De Crombrugghe and V. Rittenberg, Ann. of Phys. 151 (1983) 99

[15] M. Baake, P. Reinicke, and V. Rittenberg, J. Math. Phys. 26 (1985) 1070;

R. Flume, Ann. of Phys. 164 (1985) 189;

M. Claudson and M.B. Halpern, Nucl. Phys. B250 (1985) 689

[16] E. Bergshoeff, M.J. Duff, C.N. Pope and E. Sezgin, Phys. Lett. 199B (1987) 69;

E. Bergshoeff, E. Sezgin and Y. Tanii, ICTP preprint IC $/ 88 / 5$;

E. Bergshoeff, A. Salam, E. Sezgin and Y. Tanii, ICTP preprint IC $/ 88 / 6$

[17] E. Bergshoeff, E. Sezgin and Y. Tanii, Nucl. Phys. B298 (1988) 187

[18] P.A.M. Dirac, Lectures on quantum mechanics, Belfer Grad. School of Science Monograph Series 2, New York, 1964

[19] V.I. Arnold, Mathematical methods of classical mechanics (Springer, 1978)

[20] A. Messiah, Quantum mechanics, vols. I and II (North-Holland, 1966)

[21] Judd, Operator techniques in atomic spectroscopy (McGraw-Hill, 1963)

[22] J. Hoppe, in preparation

[23] E. Floratos and J. Iliopoulos, Phys. Lett. 201B (1988) 237

[24] R. Garreis, Karlsruhe Ph.D. Thesis, 1988

[25] E. Brézin, C. Itzykson, G. Parisi and J.-B. Zuber, Commun. Math. Phys. 59 (1978) 35

[26] M.B. Green, J.H. Schwarz and E. Witten, Superstring theory, vol. 2 (Cambridge Univ. Press, 1987)

[27] M. Günaydin and F. Gürsey, J. Math. Phys. 14 (1973) 1651

[28] B. de Wit and H. Nicolai, Nucl. Phys. B231 (1984) 506

[29] R. Courant and D. Hilbert, Methods of mathematical physics, vol. 2 (Interscience Publ., 1962)

[30] R. Slansky, Phys. Reports 79 (1981) 1

[31] A.V. Smilga, Nucl. Phys. B266 (1986) 45 